\title{
Serum proteomics links suppression of tumor immunity to ancestry and lethal prostate cancer
}

\section{Tsion Minas}

Center for Cancer Research

\section{Julián Candia}

National Cancer Institute https://orcid.org/0000-0001-5793-8989

\section{Tiffany Dorsey}

National Cancer Institute, NIH, Bethesda, MD

\section{Francine Baker}

Laboratory of Translational Genomics, Division of Cancer Epidemiology and Genetics, National Cancer Institute (NCl), National Institutes of Health (NIH) https://orcid.org/0000-0003-3133-3652

\section{Wei Tang}

$\mathrm{NCl} / \mathrm{NIH}$ https://orcid.org/0000-0002-7089-4391

\section{Maeve Kiely}

Laboratory of Human Carcinogenesis, Center for Cancer Research, National Cancer Institute ( $\mathrm{NCl})$, National Institutes of Health $(\mathrm{NIH})$

\section{Cheryl Smith}

Center for Cancer Research

\section{Symone Jordan}

Center for Cancer Research

\section{Obadi Obadi}

Laboratory of Human Carcinogenesis, Center for Cancer Research, National Cancer Institute (NCl), National Institutes of Health $(\mathrm{NIH})$

\section{Anuoluwapo Ajao}

Laboratory of Human Carcinogenesis, Center for Cancer Research, National Cancer Institute (NCl), National Institutes of Health $(\mathrm{NIH})$

\section{Yao Tettey}

University of Ghana Medical School

\section{Richard Biritwum}

University of Ghana Medical School

\section{Andrew Adjei}

University Of Ghana Medical School

James Mensah

University of Ghana Medical School

\section{Robert Hoover}


National Cancer Institute

\section{Frank Jenkins}

Hillman Cancer Center, University of Pittsburgh

\section{Rick Kittles}

City of Hope https://orcid.org/0000-0002-5004-4437

\section{Ann Hsing}

Stanford

\section{Xin Wang}

Laboratory of Human Carcinogenesis, Center for Cancer Research, National Cancer Institute (NCI), National Institutes of Health $(\mathrm{NIH})$

\section{Christopher Loffredo}

Georgetown University

\section{Clayton Yates}

Tuskegee University https://orcid.org/0000-0001-5420-9852

\section{Michael Cook}

National Cancer Institute

\section{Stefan Ambs ( $\square$ ambss@mail.nih.gov )}

Laboratory of Human Carcinogenesis, Center for Cancer Research, National Cancer Institute (NCI), National Institutes of Health (NIH) https://orcid.org/0000-0001-7651-9309

\section{Article}

Keywords: Proteomics, inflammation, prostate cancer, ancestry, survival, disparity, immune signature, African, European

Posted Date: July 13th, 2021

DOI: https://doi.org/10.21203/rs.3.rs-668276/v1

License: (c) (1) This work is licensed under a Creative Commons Attribution 4.0 International License. Read Full License

Version of Record: A version of this preprint was published at Nature Communications on April 1st, 2022. See the published version at https://doi.org/10.1038/s41467-022-29235-2. 


\section{Serum proteomics links suppression of tumor immunity to ancestry and lethal prostate cancer (13 words, 92 characters)}

Tsion Zewdu Minas ${ }^{1 \dagger}$, Julián Candia ${ }^{1 \dagger}$, Tiffany H. Dorsey ${ }^{1}$, Francine Baker ${ }^{1}$, Wei Tang ${ }^{1}$, Maeve Kiely ${ }^{1}$, Cheryl J. Smith ${ }^{1}$, Symone V. Jordan ${ }^{1}$, Obadi M. Obadi ${ }^{1}$, Anuoluwapo Ajao ${ }^{1}$, Yao Tettey ${ }^{2}$, Richard B. Biritwum ${ }^{2}$, Andrew A. Adjei ${ }^{2}$, James E. Mensah ${ }^{2}$, Robert N. Hoover ${ }^{3}$, Frank J. Jenkins ${ }^{4}$, Rick Kittles ${ }^{5}$, Ann W. Hsing ${ }^{6,7}$, Xin W. Wang ${ }^{1,8}$, Christopher A. Loffredo ${ }^{9}$, Clayton Yates ${ }^{10}$, Michael B. Cook ${ }^{3}$, and Stefan Ambs ${ }^{1 *}$

${ }^{1}$ Laboratory of Human Carcinogenesis, Center for Cancer Research, National Cancer Institute, National Institutes of Health, Bethesda, MD, USA

${ }^{2}$ University of Ghana Medical School, Accra, Ghana

${ }^{3}$ Division of Cancer Epidemiology \& Genetics, National Cancer Institute, National Institutes of Health, MD, USA

${ }^{4}$ Hillman Cancer Center, University of Pittsburgh, Pittsburgh, PA, USA

${ }^{5}$ Division of Health Equities, Department of Population Sciences, City of Hope Comprehensive Cancer Center, Duarte, CA, USA

${ }^{6}$ Stanford Cancer Institute, Stanford School of Medicine, Palo Alto, CA, USA

${ }^{7}$ Stanford Prevention Research Center, Stanford School of Medicine, Palo Alto, CA, USA

${ }^{8}$ Liver Cancer Program, Center for Cancer Research, National Cancer Institute, Natioinal Institutes of Health, Bethesda, MD, USA

${ }^{9}$ Cancer Prevention and Control Program, Lombardi Comprehensive Cancer Center, Georgetown University Medical Center, Washington, DC, USA

${ }^{10}$ Center for Cancer Research, Tuskegee University, Tuskegee, AL, USA

${ }^{\dagger}$ These authors contributed equally

Running title: Immune-oncological markers and prostate cancer disparity

Key words: Proteomics, inflammation, prostate cancer, ancestry, survival, disparity, immune signature, African, European

Abbreviations: AA, African-American; EA, European-American; OR, odds ratio; CI, confidence interval; PSA, prostate-specific antigen.

*Corresponding Author: Stefan Ambs, Laboratory of Human Carcinogenesis, National Cancer Institute, Bldg.37/Room 3050B, Bethesda, MD 20892-4258, Phone: 240-760-6836; Email: ambss@mail.nih.gov.

One sentence summary: A serum proteome-based immune function signature is upregulated in men of African ancestry and associates with lethal prostate cancer. 
Abstract (156 words):

41 There is evidence that tumor immunobiology and immunotherapy response may differ between

42 African American and European American prostate cancer patients. Here, we determined if men of

43 African descent harbor a unique systemic immune-oncological signature and measured 82

44 circulating proteins in almost 3000 Ghanaian, African American, and European American men.

45 Protein signatures for suppression of tumor immunity and chemotaxis were significantly elevated in

46 men of West African ancestry. Importantly, the suppression of tumor immunity protein signature

47 associated with metastatic and lethal prostate cancer, pointing to clinical significance. Moreover,

48 two markers, pleiotrophin and TNFRSF9, predicted poor disease survival specifically among

49 African American men. These findings indicate that immune-oncology marker profiles differ

50 between men of African and European descent. These differences may contribute to the

51 disproportionate burden of lethal prostate cancer in men of African ancestry. The elevated

52 peripheral suppression of tumor immunity may have important implication for guidance of cancer

53 therapy which could particularly benefit African American patients. 
Men of African origin bear the highest prostate cancer burden in the U.S. and globally ${ }^{1-3}$.

56 They are at an increased risk of developing fatal prostate cancer in the U.S and England ${ }^{4}$ and

57 present with more aggressive disease in the Caribbean and sub-Saharan Africa ${ }^{2,5}$. The reasons for

58 the observed global prostate cancer health disparities are unclear but may be related to an array of

59 factors such as access to health care, lifestyle and environment, and ancestral and biological

60 factors $^{6-8}$.

Previously, we and others described that tumor immunobiology differs between African-

62 American (AA) and European-American (EA) prostate cancer patients ${ }^{9-12}$. A tumor-specific immune-inflammation gene expression signature was more prevalent in prostate tumors of AA than

64 EA patients ${ }^{11}$. The occurrence of this signature in prostate tumors was associated with decreased

65 recurrence-free survival ${ }^{13}$. Furthermore, regular use of aspirin, an anti-inflammatory drug, may

66 reduce the risk of aggressive prostate cancer, disease recurrence and the lethal disease in AA

$67 \mathrm{men}^{14,15}$. Combined, these findings suggest that inflammation and host immunity may contribute to

68 prostate cancer progression but with notable differences between AA and EA men.

Ancestral factors can influence immune-related pathways ${ }^{16}$. Germline genetic variant

70 prevalence and alternative splicing in immune-inflammation-related genes can show large

71 differences amongst population groups ${ }^{17-19}$. Hence, the immune-inflammation gene expression

72 signature identified in the tumors of AA prostate cancer patients could be due to either tumor

73 biology and the associated microenvironment, ancestral factors, or systemic differences in immune-

74 oncology marker expression. In the present study, we tested the hypothesis that a distinct systemic

75 immune-inflammation signature exists in men of African ancestry that associates with prostate

76 cancer. It is the novelty of our approach that we examined the serum proteome in a large cohort of 
77 diverse men. Applying large-scale proteomics with Olink technology, we discovered the up-

78 regulation of circulating immune-oncological proteins that functionally relate to chemotaxis and

79 suppression of tumor immunity and their association with West African ancestry and lethal prostate

80 cancer. Our findings point to the clinical importance of a serum proteomic signature in prostate

81 cancer patients that may affect men of African ancestry more so than other men. 


\section{RESULTS}

Large-scale evaluation of immune-oncological proteins in the NCI-Maryland and NCI-

Ghana prostate cancer studies. To investigate if men of African descent are differentially

85

affected by a systemic immune inflammation, we utilized two case-control studies with large representations of men of African ancestry: the NCI-Ghana and NCI-Maryland Prostate Cancer Case-Control Studies. Characteristics of the participants in the two studies have been previously described $^{14,20}$. We assayed 92 circulating immune-oncological proteins in a total of 3094 serum samples containing 1505 controls and 1432 cases along with 157 randomly selected blinded duplicates. To control for any batch effects, the serum samples were assayed in a random order along with the $5 \%$ blind duplicates for intensity normalization (see Methods). Ninety-five percent of the samples passed stringent quality control leaving 1482 controls (654 Ghanaian, 374 AA, and 454 EA) and 1308 cases (489 Ghanaian, 394 AA, and 425 EA) for our analysis (table S1). The average intra- and inter-plate CV calculated based on duplicates were very low at $1.7 \%$ and $2.6 \%$, respectively. In addition, the proportion of variance explained by an inter-plate batch effect was rather minimal for each of the serum proteins even before intensity normalization (fig. S1). Out of the 92 serum proteins, 61 were detected in $100 \%$ of the samples tested and 78 were detected in $>$ $50 \%$ of the samples (fig. S2). Because 10 out of the 92 serum proteins were detected in less than $20 \%$ of the samples (fig. S2), only the remaining 82 proteins were included in our analysis (table S2). Next, we assessed how the 82 serum markers correlate with one another in Ghanaian, AA, and EA men without prostate cancer using Pearson's pairwise correlation analysis (fig. S3). The top ten observed correlations for each population group is presented in table S3. Most of these relationships have not previously been described. Most notably, epidermal growth factor levels strongly correlated with CD40L [Ghanaian $(\mathrm{r}=0.71)$, AA $(\mathrm{r}=0.83)$, and EA $(\mathrm{r}=0.80)$ men], a marker 
105 of activated T cells, whereas IL8 levels highly correlated with circulating caspase 8 in all three 106 population groups [Ghanaian $(\mathrm{r}=0.69)$, AA $(\mathrm{r}=0.82)$, and EA $(\mathrm{r}=0.80)]$.

107 Clinical and socio-demographic characteristics are associated with immune-oncological 108 proteins. Cytokine levels can be influenced by environmental exposures and disease. Therefore, 109 we investigated the association between various socio-demographic and clinical characteristics 110 (age, BMI, education, aspirin use, smoking, diabetes and PSA) with serum levels of immuno111 oncological proteins using a multivariable linear regression model (Fig. 1). We restricted this 112 analysis to the control population in the NCI-Ghana and NCI-Maryland studies to exclude the 113 potential confounding effect of prostate cancer in the analysis. Among the exposures, aspirin use 114 and blood PSA levels showed only few relationships with the profile of the 82 immune-oncology 115 markers. Other exposures and several demographics showed more robust relationships.

Aging is known to impact the immune system and is a risk factor for many diseases

117 including cancer ${ }^{21}$. In our analysis, aging was most consistently associated with the level of the 118 analytes across the three population groups, showing a significant correlation with almost half of 119 these circulating immune-oncological proteins. For example, PGF, CXCL9, Ga19, Gal1, CX3CL1, 120 TNFRSF12A, CCL23, MMP7, DCN, MMP12, CXCL13, CSF1, ADGRG1, CD4, and PTN 121 positively associated with age in all three population groups. The top-ranked biological functions 122 that associated with these age-related proteins were cell migration and positive regulation of cell 123 adhesion (fig. S4A). Age was also positively associated with lymphocyte activation, represented 124 by TNFRSF9, CRTAM, PDCD1, CD27, NCR1, TNFRSF4, KLRD1, CD83, IL12, and IL12RB1, 125 but only in the NCI-Maryland EA and AA men (fig. S4B). On the other hand, hepatocyte growth 126 factor (HGF) and vascular endothelial growth factor-A (VEGFA), two angiogenic cytokines, were 127 positively associated with age exclusively in men of African ancestry (Ghanaian and AA men). 
128 Lastly, VEGFR2, a tyrosine kinase receptor for VEGF, was negatively associated with age in EA 129 and AA men.

In contrast to the positive association of many of the immune-oncological proteins with 131 age, BMI tended to be negatively associated with these circulating immune-oncological analytes. 132 This finding may be surprising as obesity is generally thought to be associated with systemic 133 inflammation. CX3CL1 was negatively associated with BMI in all three population groups. The 134 soluble form of CX3CL1 stimulates recruitment of CX3CR1 expressing inflammatory immune 135 cells $^{22}$. CAIX and LAMP3 were inversely associated with BMI exclusively in men of African 136 ancestry, suggesting that ancestral factors may influence the relationship between BMI and 137 expression of these markers. CAIX is a hypoxia regulated metalloenzyme that exists as both 138 membrane associated and soluble form ${ }^{23}$ whose main cellular function is to catalyze the reversible 139 conversion of carbon dioxide to carbonic acid ${ }^{24}$, thereby influencing local acidity, which is known 140 to affect immune function ${ }^{25}$. LAMP3 is a member of lysosomal associated membrane glycoprotein 141 family that have a myriad of roles including lysosomal exocytosis and cholesterol homeostasis ${ }^{26}$.

142 On the contrary, serum GAL1, a glycan binding protein that mediates the suppressive function of $143 \mathrm{~T}_{\text {Reg }}$ cells $^{27}$, showed the opposite trend and was positively associated with BMI in all three 144 population groups.

145 To explore how the social/behavioral environment may affect immune-oncological serum 146 protein levels, we investigated their relationship with educational attainment. For Ghanaian men, 14727 of the 82 immuno-oncological markers were negatively associated with their education level 148 (Fig. 1). Yet only IL18 showed a significant inverse association with education for both Ghanaian 149 and AA men. Among EA men, 12 of the 82 immune-oncological proteins had significant inverse 
150 relationships with the attained level of education (Fig. 1), with some of these markers showing a 151 similar pattern among Ghanaian and EA men.

Previous studies have shown that tobacco smoking increases inflammation ${ }^{28}$. Herein, we

153

154

155

156

157

158 assessed the association between cigarette use (never, former, vs. current smoker) on the level of immune-oncological proteins in circulation. We found that current smoking was consistently associated with significantly increased level of analytes that regulate angiogenesis (ANGPT2), antigen presentation (CD83), and autophagy (LAMP3), in all three study populations (Fig. 1).

Innate immune system-driven inflammatory processes have been implicated in the pathogenesis of diabetes ${ }^{29}$. In our analysis, among the cytokines that showed an association with self-reported diabetes, serum proteins belonging to tumor necrosis factor receptor super family (CD27 and TNFRSF12A), and a matrix metalloprotease enzyme (MMP7) were positively associated with diabetes in all three population groups (Fig. 1). Others, including PGF, CX3CL1, NCR1, TNFRSF4, and TNFRSF21 were positively associated with diabetes exclusively in men with African ancestry. Functional enrichment analysis revealed that diabetes-associated CX3CL1, TNFRSF4, and TNFRSF21 are all involved in negative regulation of cytokine secretion (fig. S5). CX3CL1 is known to regulate insulin secretion ${ }^{30}$, is elevated in the serum of patients with type 2 diabetes $^{31}$, and has been implicated in diabetic nephropathy ${ }^{32}$, validating the findings in our study. C-reactive protein (CRP) is a commonly measured pro-inflammatory marker in the body and has been reported to be associated with worse prostate cancer prognosis ${ }^{33,34}$. Because it was not part of our marker panel, we measured blood CRP in 156 plasma samples from population controls of the NCI-Maryland study. Smoking was the only socio-demographic variable that had a significant association with CRP (table $\mathbf{S 4}$ ), which is consistent with the literature. Furthermore, CRP showed significant positive associations with 24 of the 82 serum proteins (TNFRSF9, IL7, 
173 PGF, IL6, Ga19, GZMH, CXCL1, TNFSF14, Gal1, PDL1, HGF, HO1, CD70, TNFRSF12A,

174 CCL3, MMP7, ANGPT2, VEGFA, CCL20, KLRD1, CSF1, CD4, MCP3, and CXCL11).

175 The systemic immune-oncological cytokine profile in men of African ancestry is distinct from

176 men of European ancestry. To investigate if ancestral population group differences may

177 influence circulating levels of the immune-oncological markers, we performed an unsupervised

178 clustering analysis examining how the levels of the 82 immune-oncological analytes would group

179 men without prostate cancer from Ghana and the US. Notably, these analytes tended to cluster by

180 population group, with levels in Ghanaian men being most distant from EA men while AA samples

181

tended to cluster in between these two groups (Fig. 2), suggesting that the ancestral background

182 may have a significant impact on this immune-oncological protein profile.

To further evaluate the influence of ancestry, we estimated West African ancestry in AA

and EA population controls of the NCI-Maryland study and its relationship with the cytokine

185 profile. West African ancestry was determined using 100 validated ancestry informative markers

$186{ }^{35}$. The approach showed that, to some extent, the variance in the levels of several immune-

187 oncological analytes is strongly influenced by the degree of West African ancestry of these

188 individuals (Fig. 3A). The variance in 45 of the analytes were significantly $(P<0.05)$ influenced

189 by degree of West African ancestry (table S5). The levels of 42 analytes were significantly

190 accounted for by West African ancestry even after adjusting for age, BMI, aspirin use, education,

191 income, diabetes, and smoking status (table S6). CXCL5, CXCL1, MCP2, MCP1, CXCL11,

192 CCL23, PTN, TWEAK, NCR1, IL18 and CCL17 were the top-ranked proteins (tables S5-S6).

193 Adjusting the significance threshold by Bonferroni $\left(\mathrm{P}_{\mathrm{B}}=0.05 / 82=0.00061\right)$, which is the most

194 stringent criterion to adjust for multiple testing, the relationship of the top 28 proteins with West

195 African ancestry remained significant. For instance, $41 \%$ and $50 \%$ of the variance in the serum 
196 levels of CXCL1 and CXCL5, respectively, was accounted for by the degree of West African 197 ancestry (Fig. 3A and tables S5-S6). When we compared the levels of these proteins across the 3 198 population groups, we observed a significant African ancestry-related trend (Fig. 3B), with 12 of 199 the 82 circulating immune-oncological proteins (CXCL5, CXCL1, CXCL11, MCP2, CCL17, 200 MCP4, CD70, MMP12, PDL2, MMP7, CCL19, and ANGPT2) being significantly elevated in 201 both Ghanaian and AA men compared to EA men (table S7); twelve other markers (MCP1, IL12, 202 CCL23, CD8A, NCR1, TNFRSF4, TNFSF14, TWEAK, IL7, HGF, HO1, TNFRSF21, and ANG1) 203 were inversely related to West African ancestry (table S8).

204 Cytokines associated with suppression of tumor immunity and chemotaxis are upregulated 205 in men of African ancestry. Levels of many of the 82 immune-oncology markers showed a 206 marked association with ancestry. To better define the functional implications of these 207 population group differences, we grouped the 82 proteins into six biological processes according 208 to Olink guidelines (table S9): apoptosis/cell killing, autophagy/metabolism, 209 chemotaxis/trafficking to tumor, suppression of tumor immunity (Th2 response, tolerogenic), 210 promotion of tumor immunity (Th1 responses), or vasculature and tissue remodeling. To gain 211 insight on how activation of these six processes/pathways may differ by population group, we 212 compared process/pathway sum scores between Ghanaian, AA, and EA men without prostate 213 cancer. Of these pathways, chemotaxis, promotion of tumor immunity, and suppression of tumor 214 immunity were significantly different in their predicted activity between AA and EA men (Fig. 215 4). AA men had significantly higher scores for chemotaxis and suppression of tumor immunity 216 when compared to EA men, indicating higher activity in AA men, but a lower score for 217 promotion of tumor immunity. Ghanaian men had even higher scores for chemotaxis and 218 suppression of tumor immunity than both AA and EA men (Fig. 4C and E), indicating a 
possible association with West African ancestry. The latter was corroborated with our finding that the chemotaxis and suppression of tumor immunity scores positively correlated with the proportion of West African ancestry within the NCI-Maryland cohort (Spearman's rho=0.23, $P$ $<0.001$, for chemotaxis score; Spearman's rho $=0.15, P<0.001$, for suppression of immunity score). Even though apoptosis and vasculature-associated cytokines were not significantly different between EA and AA men, we found both processes to be elevated in the Ghanaian men.

\section{Suppression of tumor immunity is associated with reduced survival of prostate cancer} patients. Next, we examined the clinical implication of our findings and assessed the association of pathway activity with survival of prostate cancer cases or controls in the NCI-Maryland study. As of the end of 2018, out of the 819 cases, there have been 202 deaths in our case population, of whom $103(51 \%)$ had a cancer diagnosis as the recorded primary cause of death, and $28 \%$ of all deaths $(n=57)$ were directly attributed to prostate cancer. On the other hand, 99 of the 828 population controls had died by the end of 2018. Median survival follow-up for cases and controls were 8.6 and 6.7 years, respectively. With these data, we built a multivariable Cox regression model with all biological processes/pathways and adjustment for other covariables (see Methods). Among the six defined pathways, only suppression of tumor immunity showed independent association with survival of cases (Fig. 5). Prostate cancer patients with an increased activity of this pathway had the highest risk of death from all causes (Fig. 5A, table S10). Moreover, prostate cancer patients with elevated suppression of tumor immunity at diagnosis had the highest risk of prostate cancer-specific mortality, albeit marginally significant $(P=0.057)$ (Fig. 5B, table S11). Notably, suppression of tumor immunity was not associated with all-cause mortality of population controls (table S12), suggesting that the association with all-cause mortality among cases might be prostate cancer-related. Lastly, prostate cancer 
242 patients with increased suppression of tumor immunity were also significantly more likely to die

243 from any cancer (prostate cancer or secondary cancer) following the prostate cancer diagnosis

244 (Fig. 5C, table S13), indicating a more general predisposition to cancer in patients with a high

245 suppression of tumor immunity score.

246 Elevated suppression of tumor immunity is associated with metastatic prostate cancer. To

247 further corroborate the significance of suppression of tumor immunity in the development of

248 lethal prostate cancer, we assessed its association with prostate cancer aggressiveness per NCCN

249 guidelines (see Methods). Information on TNM stage was only obtainable for the NCI-Maryland

250 prostate cancer patients, hence only these cases were scored according to the NCCN guidelines.

251 Patients with a high suppression of tumor immunity score were at substantially increased odds of 252 being diagnosed with regional or distant metastasis (HR 3.79, 95\% CI 1.59-9.04, > median vs. $\leq$

253 median) (Table 1), consistent with the disease survival data. The data showed a significant trend 254 in the association of elevated suppression of tumor immunity with disease aggressiveness $(P$ 255 trend=0.004) (Table 1). Stratified analysis by race/ethnicity revealed that high suppression of 256 tumor immunity was associated with metastatic prostate cancer more so among AA than EA 257 men.

258 Blood levels of TNFRSF9 and pleiotrophin (PTN) predict lethal prostate cancer among AA

259 men. To identify individual drivers of the relationship between immune-oncology markers and

260 lethal prostate cancer, we applied a cross-validated, regularized Cox regression model using

261 eNetXplorer (see Methods). Included in this model were the 82 immune-oncology markers and

2626 covariates of clinical significance (age, education, BMI, smoking history, aspirin use, and

263 diabetes). Lasso regression (alpha=1) was selected based on overall performance (fig. S6).

264 Utilizing this method, we could not identify a robust predictive signature of lethal prostate cancer 
265 for EA patients. However, for AA patients, a signature primarily driven by TNFRSF9 and PTN 266 (both positively associated with the risk of lethal disease) and regular aspirin use (negatively 267 associated with risk) were the top predictors $(P<0.05)$ based on two selection criteria: the 268 feature frequency (Fig. 6A) and the weight of the features' contribution to the prediction (Fig. 269 6B). These features combined predicted prostate cancer-specific mortality with an accuracy of $27083.7 \%(\mathrm{SE}=3.8 \%)$. Our finding that regular aspirin use was a predictor of improved survival 271 among AA men is consistent with previously published data from this case-control study ${ }^{14}$ and 272 the Southern Community Cohort Study ${ }^{15}$. The two proteins alone, TNFRSF9 and PTN, predicted 273 prostate cancer-specific mortality with 78.2\% (SE=4.2\%) accuracy. AA prostate cancer patients 274 with high levels ( $>$ median) of both TNFRSF9 and PTN in their blood at diagnosis had the worst 275 prostate cancer-specific survival (Fig. 6C). By 10 years, 33\% of cases with high levels of both 276 TNFRSF9 and PTN died of prostate cancer compared to only 5\% of cases with low levels of 277 both or either of these proteins (Fig. 6C), highlighting the utility of these blood markers for risk 278 stratification of AA prostate cancer patients. 


\section{DISCUSSION}

In this study, we describe consistent differences in the expression of immune and chemotaxis-related markers in men from three population groups, with two of them - AA and

282 Ghanaian men - having an ancestral relationship due to the trans-Atlantic slave trade. Most

283 notably, expression of immune-oncology markers related to immune suppression were up-

284 regulated in men of West African ancestry and were associated with lethal prostate cancer. While ancestry can explain some of the observations, other and yet unknown factors may contribute to these clinically significant differences in immune function and chemotaxis.

Infections endemic to certain regions have shaped the immune response in affected

288 populations, leaving a lasting genetic and epigenetic footprint ${ }^{36}$. As such, population differences

289 in exposures to fatal pathogens have led to population heterogeneity in the immunome. It has

290 been estimated that as many as 360 immune-related genes have been targets of positive selection 291 and have functional variations between populations ${ }^{37}$. Consistent with these observations, we 292 now report population differences in circulating immune-oncological proteins among Ghanaian, 293 AA, and EA men. We found that the serum proteome-defined immunome of Ghanaian men 294 resembles the immunome of AA men more so than EA men. We identified CXCL5, CXCL1, 295 MCP2, MCP1, and CXCL11 as the top immune-oncological proteins associated with West 296 African ancestry. Four of these chemokines (CXCL5, CXCL1, MCP1, and CXCL11) are targets 297 of Duffy Antigen Receptor for Chemokines (DARC) binding ${ }^{38}$. DARC is a non-signaling 298 receptor that binds to both CXC and CC family of chemokines and acts as a depot for 299 chemokines on erythrocytes and as decoy receptor on endothelial cells ${ }^{39}$. DARC expression 300 modulates the susceptibility to clinical Plasmodium vivax malaria and loss of its expression on 301 erythrocytes, which frequently occurs in sub-Saharan African populations due to germline 
302 genetic variants, confers resistance against malarial infection ${ }^{40}$. Its loss may also influence cancer

303 susceptibility ${ }^{41,42}$. Consequently, these individuals lack the ability to sequester the target

304 chemokines, leading to elevated concentration of the chemokines in circulation ${ }^{43}$. Accordingly,

305 we found that CXCL5, CXCL1, and CXCL11 were 2-3-fold higher in sera of Ghanaian or AA

306 men than EA men. Given the angiogenic properties of these chemokines ${ }^{44}$, their role in cancer

307 progression has been proposed ${ }^{45}$.

As a key finding, we report that serum proteins driving chemotaxis and suppression of

309 tumor immunity were elevated in men of African ancestry, suggesting persistent population

310 differences in stimulation of leukocyte recruitment and T cell mediated immune response. Such

311 differences may predispose men of African descent to a distinct tumor microenvironment.

312 Although the direct impact of the peripheral immunome on the prostate tumor microenvironment

313 requires further investigation, we and others have previously reported stark differences in the

314 immune landscape of prostate tumors of AA men as compared to EA men ${ }^{9-13}$. For instance,

315 programmed cell death ligand-1 (PD-L1), which suppresses T cell-mediated tumor immunity,

316 was found to be overexpressed in AA prostate tumors ${ }^{46}$. Recent work by Awasthi et al. reported

317 that AA prostate tumors tend to be enriched for immune pathways that are associated with poor

318 clinical outcomes ${ }^{47}$. We show with our current work that elevated, peripheral suppression of

319 tumor immunity associates with lethal prostate cancer. Hence, population differences in

320 suppression of tumor immunity may contribute to the disproportionate burden of lethal prostate

321 cancer among men of African ancestry. On the other hand, such differences may offer a

322 therapeutic advantage for immunotherapeutic strategies that are tailored to target immune

323 suppressive pathways. A recent study provided a first indication that differences in the response

324 to cancer vaccines may lead to higher survival rates among AA men ${ }^{48}$. 

slow-growing disease remains a major challenge in clinical oncology. Risk stratification

327 strategies are particularly needed for AA prostate cancer patients who disproportionately bear the 328 prostate cancer burden. This study identified TNFRSF9 and PTN as candidate predictive blood 329 markers for prostate cancer mortality among AA patients. AA patients with high levels of both 330 TNFRSF9 and PTN in their sera had the highest risk of dying from prostate cancer. The membrane form of TNFRSF9 possesses antitumor properties and agonistic anti-TNFRSF9

332 antibodies are currently in clinical trials ${ }^{49,50}$. On the contrary, the soluble isoform of TNFRSF9 333 that we measured, generated by alternative splicing ${ }^{51}$, has been shown to antagonize antitumor 334 immune response hence promote tumor survival most likely by acting as decoy receptor ${ }^{52,53}$. 335 Regulatory T cells described as Tregs are thought to be a major source of secreted TNFRSF9 ${ }^{54,55}$. 336 Recently, TNFRSF9 mRNA level was shown to be a robust marker of tumor-infiltrating Tregs 337 that suppress antitumor response ${ }^{56}$. Moreover, high numbers of TNFRSF9-expressing Tregs were associated with poor survival outcomes across multiple human cancers ${ }^{56}$, consistent with 339 our findings that serum TNFRSF9 associates with lethal prostate cancer. Although pleiotrophin, 340 the second protein marker associated with lethal prostate cancer in AA men, may not have the 341 same immune function that soluble TNFRSF9 exhibits, it is a secreted cytokine with important 342 roles in promoting angiogenesis and metastasis ${ }^{57}$. Recently, pleiotrophin was described as a 343 serum-based biomarker of pro-metastatic prostate cancer ${ }^{58}$, consistent with our findings in this 344 study.

To the best of our knowledge, this is the first study with a large representation of men of 346 African descent who contributed to immune-oncological proteome profiling. With the advent of 347 increasing immunotherapies in the drug development pipeline, such studies may inform research 
348 on population differences in the immune landscape that need to be considered when designing 349 therapies that exploit the immune response. Furthermore, our study may provide unique insights 350 into variations in the manifestation and pathogenesis of different immune related diseases among 351 different population groups.

352 In conclusion, it is a key finding of our study that suppression of tumor immunity was

353 increased in Ghanaian and AA men, when compared to EA men, and associates with lethal 354 prostate cancer. As such, these findings provide a novel insight into potential causes of the 355 prostate cancer health disparity. 
NCI-Maryland prostate cancer case-control study. This study and the eligibility criteria have been previously described ${ }^{14,59}$. Race/ethnicity was assigned based on self-identification as either black or AA or as Caucasian or EA. The study was initiated to test the primary hypothesis that environmental exposures and ancestry-related factors contribute to the excessive prostate cancer burden among AA men. The study was approved by the NCI (protocol \# 05-C-N021) and the University of Maryland (protocol \#0298229) Institutional Review Boards and all 364 participants signed an informed consent. Cases were recruited at the Baltimore Veterans Affairs 365 Medical Center and the University of Maryland Medical Center. A total of 976 cases (489 AA and 487 EA men) were recruited into this study between 2005 and 2015. Controls were

367 identified through the Maryland Department of Motor Vehicle Administration database and were frequency-matched to cases on age and race. A total of 1,034 population controls were recruited (486 AA and 548 EA men). At the time of enrollment, both cases and controls were administered a survey by a trained interviewer and a blood sample was collected. Serum

371 samples were available for 846 cases (407 AA and 439 EA) and 846 controls (382 AA and 464

372 EA), therefore only these individuals were used for the study herein. Most of the 846 cases

$373(85 \%)$ were recruited within a year of the disease diagnosis with a median of 5.1 months 374 between disease diagnosis and blood collection.

375 NCI-Ghana prostate cancer case-control study. This case-control study has been previously 376 described $^{20}$. The study was designed to study lifestyle, environmental, and genetic risk factors 377 for prostate cancer in African men. The study was approved by institutional review boards at 378 the University of Ghana (protocol \#001/01-02) and at the National Cancer Institute (protocol $379 \#$ 02CN240). Prior to study enrollment, all participants signed an informed consent. Prostate 
cancer cases were recruited at Korle Bu Teaching Hospital in Accra, Ghana between 2008 and 2012. The cases were diagnosed using Digital Rectal Exam (DRE) and PSA tests, followed by biopsy confirmation. Immediately after diagnosis and before treatment, cases were consented and asked to submit blood specimen and questionnaire data. Controls were identified through probability sampling using the 2000 Ghana Population and Housing Census data to recruit approximately 1,000 men aged 50-74 years in the Greater Accra region between 2004 and 2006. These men were confirmed to not have prostate cancer by PSA testing and DRE. Serum samples were available for 586 prostate cancer cases and 659 population controls; hence, only these individuals were used for the study herein.

Serum sample processing. The participants in the two studies provided blood samples at time of recruitment. For the NCI-Maryland study, most blood samples were processed the same day, but always within 48 hours, after storage in a refrigerator. For the NCI-Ghana study, blood samples were processed within 6 hours. In this study, population controls provided fasting blood. Serum was prepared using standard procedures and aliquots were stored at $-80^{\circ} \mathrm{C}$. Serum samples were shipped from Ghana to the NCI in dry ice boxes.

Serum protein measurement. Serum levels of 92 immuno-oncology panel proteins were measured simultaneously using a proprietary multiplex Proximal Extension Assay (PEA) by Olink Proteomics (Boston). Olink utilizes a relative quantification unit, Normalized Protein eXpression (NPX), which is in a Log2-format. Serum samples from NCI-MD study (846 cases and 846 controls) and NCI-Ghana study (586 cases and 659 controls) were completely randomized and were assayed in that order. In addition to the built-in internal controls, $5 \%$ blinded duplicates were randomly selected and were randomized along with the original set of samples. Protein levels were intensity normalized to adjust for batch effect. Because all our 
samples were randomized across plates, a global adjustment was used to center the values for each assay around its median and across all plates. Ninety-five percent of the samples passed a stringent quality control (NCI-MD study: 819 cases and 828 controls; NCI-Ghana study: 489 cases and 654 controls) - with coefficients of variation (CV) among duplicates at $<10 \%$ for every marker. Out of the 92 proteins assayed, IL33, IL35, IL21, IL2, IFN $\beta$, IL13, IL1 $\alpha$, CXCL12, IFN $\gamma$, and TNF were detected in less than $20 \%$ of the samples, hence the remaining 82 proteins were used for subsequent analysis (table S2).

Functional annotation and biological processes scores. Proteins were grouped into six biological processes based on their respective biological roles following the Olink guideline: apoptosis/cell killing, autophagy/metabolism, chemotaxis/trafficking to tumor, suppression of tumor immunity (Th2 response, tolerogenic), promotion of tumor immunity (Th1 responses), or vasculature and tissue remodeling. Apoptosis, autophagy, chemotaxis, suppression of tumor immunity, promotion of tumor immunity, or vasculature scores were calculated for each study participant as the mean z-score value for the proteins belonging to the respective biological process. For survival analysis, the biological process/pathway scores were evaluated as continuous variables. To evaluate the association of suppression of tumor immunity with aggressive prostate cancer, we grouped suppression of tumor immunity scores into low ( $\leq$ median) and high ( $>$ median) with cutoffs determined using the distribution of the score among population controls of the NCI-Maryland study.

Prostate Specific Antigen (PSA) measurement. For the cases in the NCI-Maryland cohort, PSA levels were obtained from medical record. For the controls of the NCI-Maryland study, total PSA was measured from stored serum aliquots using the human total PSA ELISA Kit (Abcam, ab188388). About 7\% $(n=56)$ of the controls in the NCI-Maryland cohort had PSA greater than 
$2.5 \mathrm{ng} / \mathrm{ml}$, while only $3 \%(\mathrm{n}=27)$ had blood PSA over $4 \mathrm{ng} / \mathrm{ml}$. For the controls in the NCI-

Ghana study, close to $20 \%(\mathrm{n}=132)$ had a PSA greater than $2.5 \mathrm{ng} / \mathrm{ml}$, while about $11 \%(\mathrm{n}=73)$ had PSA over $4 \mathrm{ng} / \mathrm{ml}$.

C-reactive protein (CRP) measurement. Plasma CRP was assayed using an ELISA assay (cat\# ab99995, Abcam, United States) according to the manufacturer's instructions. Two microliters of plasma samples were added to $398 \mu \mathrm{L}$ of 1x Diluent D, followed by a second 1: 200 dilution steps for each sample. One-hundred microliters of CRP standard (0 to $600 \mathrm{pg} / \mathrm{mL}$ ) and the diluted samples were loaded as duplicates into pre-coated 96-well plates. Samples were incubated overnight at $4^{\circ} \mathrm{C}$ with gentle shaking, followed by incubations with the anti-human CRP antibody and the horseradish peroxidase-streptavidin solution. CRP was quantified measuring absorbance at $450 \mathrm{~nm}$ with a microplate reader.

\section{West African ancestry estimation for participants in the NCI-Maryland case-control study.}

Genomic DNA was isolated from buffy coats (DNeasy Blood \& Tissue Kit - Qiagen) or mouthwash samples (standard phenol-chloroform technique). Isolated DNA was genotyped for 100 ancestry informative markers using the Sequenom MassARRAY iPLEX platform, as previously described ${ }^{35}$. Single nucleotide polymorphism genotype calls were generated using Sequenom TYPER software. A genotype concordance rate of $>99 \%$ was observed for all markers. Admixture estimates for each study participant were calculated using a model-based clustering method as implemented in the program STRUCTURE v2.3. We applied STRUCTURE v2.3 with an admixture model estimating K (number of sub populations) from 2 to 5 with 100 iterations and parental population genotypes from West Africans, Europeans, and Native Americans, yielding three admixture estimations (West African, European, Native American). For a subset (83\%) of the cases and controls, additional West African ancestry 
449 estimates were provided by the Cancer Genomics Research Laboratory/NCI-Leidos from a

450 genome-wide association study using the Infinium HumanOmni5-Quad BeadChip array. West

451 African ancestry estimates using the two approaches were very similar $(\mathrm{r}=0.98)$.

452 Association of clinical/socio-demographic characteristics with immune-oncological

453 proteins. The association of age, body mass index (BMI), education, aspirin use, smoking,

454 diabetes, and PSA levels with the relative abundance of individual analytes was assessed by

455 means of multivariable linear regression models implemented by the function $1 \mathrm{~m}$ in the base

$456 \mathrm{R}$ package stats (version 3.6.1). These variables were chosen because they have either been

457 linked to prostate cancer risk and survival or may influence the status of inflammation and

458 host immunity. An analyte (as response variable) was considered significantly associated

459 with clinical and socio-demographic covariables if the multivariable model yielded $P<0.05$

460 on the F-statistic. If this condition was satisfied, the association between the target analyte

461 and each individual covariable was characterized by the corresponding $P$ value and

462 coefficient.

463 Analysis of variance. Variance analysis for the levels of each of the 82 immune-oncological

464 cytokines were simultaneously assessed as a function of genetic estimation of West African

465 admixture among men without prostate cancer from the NCI-Maryland study. The analysis

466 was implemented by the function aov in the base R package stats (version 3.6.1).

467 Heatmap plots. Heat map plots were generated using Broad Institute's web-based matrix

468 visualization and analysis platform - Morpheus (https://software.broadinstitute.org/morpheus).

469 To avoid spurious effects from outliers in heatmap plots, each protein's range of abundance

470 values were set to saturate at the $1^{\text {st }}$ and $99^{\text {th }}$ percentiles. To account for widely different 
471 abundance ranges for different proteins in the assay, each protein's measured abundances across

472 the cohort were Z-score transformed.

473 Gene ontology (GO) enrichment analysis. GO terms with an enrichment in proteins of interest 474 were identified using Over-Representation Analysis (ORA) as part of the web tool WebGestalt 475 (WEB-based Gene SeT AnaLysis Toolkit). Enriched gene sets were further processed using 476 affinity propagation (R package apcluster) to cluster gene sets according to functional similarity.

477 Survival analysis. Information on patient survival was only obtainable for the NCI-Maryland 478 prostate cancer patients. Survival data was obtained from the National Death Index (NDI) 479 database. We calculated survival for cases from date of diagnosis to either date of death or to the 480 censor date of December 31, 2018. We built a multivariable Cox regression model with all 481 482 483 and prostate cancer-specific mortality of cases. We adjusted for the following potential 484 confounding factors: age at study entry (years), body-mass index (BMI, $\mathrm{kg} / \mathrm{m}^{2}$ ), self-reported 485 race (AA/EA), education (high school or less, some college, college, professional school), 486 income (less than $\$ 10 \mathrm{k}, \$ 10-30 \mathrm{~K}, \$ 30-60 \mathrm{~K}, \$ 60-90 \mathrm{k}$, greater than $\$ 90 \mathrm{k}$ ), smoking history 487 (never, former, current), diabetes (no/yes), aspirin use (no/yes), and treatment $(0=$ none, $4881=$ surgery, $2=$ radiotherapy, $3=$ hormone, $4=$ combination $)$. Missing values for education $(n=1)$, 489 smoking history $(n=5)$, and income $(n=63)$ were imputed using the R package missForest, which 490 implements nonparametric missing value imputation based on random forests. In the overall 491 survival analysis of population controls, we calculated survival from the date of interview to 492 either date of death or to the censor date of December 31st, 2018. We applied the Cox regression 493 model to estimate adjusted HR and 95\% CI and adjusted for all the confounding factors listed 
494 above except for treatment. Missing values for education $(\mathrm{n}=1)$, smoking history $(\mathrm{n}=7)$, and 495 income $(n=67)$ were imputed using the R package missForest. The reported HRs indicate the 496 change in risk of dying when the biological process z-score value increases by 1 while holding

497 all the other biological processes' z-scores and covariates constant.

Classification of cases using National Comprehensive Cancer Network (NCCN) Risk Score.

499 Cases were assigned to risk groups based on the patients' TNM stage, Gleason score, Gleason

500 pattern, and PSA level at diagnosis according to the 2019 NCCN guideline for prostate cancer ${ }^{60}$.

501 Information on TNM stage was only obtainable for the NCI-Maryland prostate cancer patients,

502 hence only these cases were scored. Cases were categorized as localized, regional, and

503 metastatic prostate cancer based on their clinical parameters at the time of diagnosis. Localized

504 prostate cancer cases were further classified into low, intermediate, high, and very high risk

505 based on the likelihood of their disease to progress to lethal prostate cancer per the $2019 \mathrm{NCCN}$

506 guideline ${ }^{60}$. Prostate cancer cases with lymph node involvement but no distant metastasis at

507 diagnosis were classified as regional prostate cancer while those with distant metastasis at the

508 time of diagnosis were classified as metastatic prostate cancer. For our analysis, we condensed

509 these risk groups into 4 categories (low, intermediate, high/very high, and regional/metastatic).

510 Developing a predictive proteomic signature of lethal prostate cancer. The analysis was

511 restricted to the cases from NCI-Maryland study for whom we had survival data. We stratified by

512 race/ethnicity into AA cases (360 censored, 34 prostate cancer deaths) and EA cases (402

513 censored, 23 prostate cancer deaths). To identify a multi-analyte proteomic signature that is

514 predictive of lethal prostate cancer, 88 features were evaluated [82 immune-oncological proteins

515 along with six demographic/clinical variables (education, age, BMI (BMI, $\mathrm{kg} / \mathrm{m}^{2}$ ), smoking

516 history, diabetes, and aspirin use)]. Missing values for education $(\mathrm{n}=1)$ and smoking history 
$517(\mathrm{n}=5)$ were imputed using R package missForest. R package eNetXplorer (version 1.1.2 $)^{61}$ was

518 implemented to build cross-validated, regularized Cox regression models with different elastic

519 net mixture parameters from ridge $(\mathrm{alpha}=0)$ to lasso $(\mathrm{alpha}=1)$. Alpha was selected based on

520 overall performance assessed as a function of the 5-fold cross-validated quality function

521 (concordance) and the empirical $P$ value generated from comparing the model against a

522 statistical ensemble of null models created by random permutations of the response (i.e. survival

523 time/status randomized across subjects in the cohort). These results comprise 10,000 Cox

524 regression elastic net realizations arising from 200 randomly generated folds, each of them

525 compared against 50 null model permutations. Features' performance as predictors was

526 evaluated using two different, but complementary selection criteria: feature coefficients and

527 feature frequencies. The feature frequency measure captures the significance of how often a

528 feature is chosen in an in-bag model. When it is chosen, the feature coefficient measure captures

529 the significance of the feature's weight in the in-bag model. See the publication by Candia et al

530 for more details on this method ${ }^{61}$. Using only the significant protein features from both selection

531 criteria, a multivariate Cox regression model was run. Risk stratification was used to generate

532 Kaplan-Meier plots and log-rank tests of significance.

533 Statistical analysis. Data analyses were performed using Stata/SE 16.0 and R statistical software

534 packages. All statistical tests were two-sided, and an association was considered statistically

535 significant with $P<0.05$.

536

537 


\section{Supplementary Materials:}

Fig. S1. Variance explained by the inter-plate batch effect

Fig. S2. Detection frequencies of 92 immuno-oncological markers measured in 2937 serum samples

Fig. S3. Correlation matrix presenting Pearson pairwise correlations for each of the 82 serum protein pairs

Fig. S4. Gene ontology (GO) enrichment analysis

Fig. S5. Functional enrichment analysis of proteins positvely associated with diabetes in men of African ancestry

Fig. S6. Performance of regularized cox regression models across alpha

Table S1. Characteristics of prostate cancer cases and population controls of the NCI-Maryland and NCI-Ghana Study

Table S2. List of 82 Immuno-oncological proteins detected in more than $20 \%$ of theserum samples

Table S3. Top 10 Pearson pairwise correlations in men without prostate cancer

Table S4. The association of blood CRP with clinical/sociodemographic variables estimated using multiple linear regression

Table S5. The fraction of variance in each of the serum proteins explained by degree of West African ancestry

Table S6. The fraction of variance in each of the serum proteins explained by degree of West African ancestry after adjusting for difference in age, bmi, aspirin use, education, diabetes status, smoking, and income

Table S7. Immune oncological markers that are significantly elevated in both Af and AA men compared to EA men

Table S8. Immune oncological markers that are significantly downregulated in both Af and AA men compared to EA men

Table S9. Serum proteins grouped by biological process

Table S10. Effect of biological processes scores on all-cause mortality of prostate cancer patients Table S11. Effect of biological processes scores on prostate cancer-specific mortality Table S12. Effect of biological processes scores on all-cause mortality of population controls Table S13. Effect of biological processes scores on mortality from any cancer following a diagnosis with prostate cancer

Acknowledgments: We would like to thank personnel at the University of Maryland and the Baltimore Veterans Administration Hospital for their contributions with the recruitment of participants to the NCI-Maryland study. We would also like to thank Prof. Edward D. Yeboah as the original Ghana PI and Ms. Evelyn Tay as the original Study Manager for the NCI-Ghana study.

\section{Funding:}

DoD award W81XWH1810588 (to SA, CY)

U54 CA118623- CY (NCI) and U54-MD007585-26-CY (NIMHD) (to CY)

Intramural Research Program of the NIH, National Cancer Institute (NCI), Center for Cancer Research and Division of Cancer Epidemiology and Genetics (to SA, MBC) 
584 Author contributions:

585 Conceptualization: TZM, CY, MBC, SA

586 Data curation: TZM, THD, MK, CJS, SVJ, OMO, AA, FJJ, RK

587 Formal Analysis: TZM, JC, RK

588 Funding acquisition: $\mathrm{CY}, \mathrm{MBC}, \mathrm{SA}$

589 Investigation: TZM, JC, FJJ

590 Methodology: TZM, JC, CAL, MBC, SA

591 Project administration: THD, FB

592 Resources: WT, YT, RBB, AAA, JEM, RNH, AWH, MBC, SA

593 Supervision: WT, SA

594 Visualization: TZM, JC

595 Writing - original draft: TZM

596 Writing - review \& editing: TZM, FB, WT, MK, CJS, YT, RBB, AAA, JEM, XWW, CAL, CY,

597 MBC, SA

598

599 Conflicts of interest: The authors declare that they have no competing interests.

600

601 Data and materials availability: All data are available in the main text or the supplementary

602 materials. 


\section{REFERENCES}

1 Powell, I. J. Epidemiology and pathophysiology of prostate cancer in African-American men. J Urol. 177, 444-449 (2007).

2 Rebbeck, T. R. et al. Global patterns of prostate cancer incidence, aggressiveness, and mortality in men of african descent. Prostate Cancer 2013, 560857 (2013).

3 Culp, M. B., Soerjomataram, I., Efstathiou, J. A., Bray, F. \& Jemal, A. Recent Global Patterns in Prostate Cancer Incidence and Mortality Rates. Eur Urol 77, 38-52, doi:10.1016/j.eururo.2019.08.005 (2020).

4 Butler, E. N., Kelly, S. P., Coupland, V. H., Rosenberg, P. S. \& Cook, M. B. Fatal prostate cancer incidence trends in the United States and England by race, stage, and treatment. Br J Cancer 123, 487-494, doi:10.1038/s41416-020-0859-x (2020).

5 Heyns, C. F., Fisher, M., Lecuona, A. \& van der Merwe, A. Prostate cancer among different racial groups in the Western Cape: presenting features and management. $S$ Afr Med J 101, 267-270, doi:10.7196/samj.4420 (2011).

6 Wallace, T. A., Martin, D. N. \& Ambs, S. Interactions among genes, tumor biology and the environment in cancer health disparities: examining the evidence on a national and global scale. Carcinogenesis 32, 1107-1121 (2011).

7 Rebbeck, T. R. Prostate Cancer Disparities by Race and Ethnicity: From Nucleotide to Neighborhood. Cold Spring Harb Perspect Med 8, doi:10.1101/cshperspect.a030387 (2018).

8 Lachance, J. et al. Genetic Hitchhiking and Population Bottlenecks Contribute to Prostate Cancer Disparities in Men of African Descent. Cancer Res 78, 2432-2443, doi:10.1158/0008-5472.CAN-17-1550 (2018).

9 Hardiman, G. et al. Systems analysis of the prostate transcriptome in African-American men compared with European-American men. Pharmacogenomics 17, 1129-1143, doi:10.2217/pgs-2016-0025 (2016).

10 Powell, I. J. et al. Genes associated with prostate cancer are differentially expressed in African American and European American men. Cancer Epidemiol Biomarkers Prev 22, 891-897, doi:10.1158/1055-9965.EPI-12-1238 (2013).

11 Wallace, T. A. et al. Tumor immunobiological differences in prostate cancer between African-American and European-American men. Cancer Res 68, 927-936, doi:10.1158/0008-5472.CAN-07-2608 (2008).

12 Yuan, J. et al. Integrative comparison of the genomic and transcriptomic landscape between prostate cancer patients of predominantly African or European genetic ancestry. PLoS Genet 16, e1008641, doi:10.1371/journal.pgen.1008641 (2020).

13 Tang, W. et al. IFNL4-DeltaG Allele Is Associated with an Interferon Signature in Tumors and Survival of African-American Men with Prostate Cancer. Clin Cancer Res 24, 5471-5481, doi:10.1158/1078-0432.CCR-18-1060 (2018).

14 Smith, C. J. et al. Aspirin Use Reduces the Risk of Aggressive Prostate Cancer and Disease Recurrence in African-American Men. Cancer Epidemiol Biomarkers Prev 26, 845-853, doi:10.1158/1055-9965.EPI-16-1027 (2017).

15 Tang, W. et al. Aspirin Use and Prostate Cancer among African American Men in the Southern Community Cohort Study. Cancer Epidemiol Biomarkers Prev, doi:10.1158/1055-9965.EPI-19-0792 (2020). 
64716 Nedelec, Y. et al. Genetic Ancestry and Natural Selection Drive Population Differences 648 in Immune Responses to Pathogens. Cell 167, 657-669 e621, doi:10.1016/j.cell.2016.09.025 (2016).

651 Ness, R. B., Haggerty, C. L., Harger, G. \& Ferrell, R. Differential distribution of allelic variants in cytokine genes among African Americans and White Americans. Am J Epidemiol 160, 1033-1038, doi:10.1093/aje/kwh325 (2004).

18 Rotival, M., Quach, H. \& Quintana-Murci, L. Defining the genetic and evolutionary architecture of alternative splicing in response to infection. Nat Commun 10, 1671, doi:10.1038/s41467-019-09689-7 (2019). Van Dyke, A. L., Cote, M. L., Wenzlaff, A. S., Land, S. \& Schwartz, A. G. Cytokine SNPs: Comparison of allele frequencies by race and implications for future studies. Cytokine 46, 236-244, doi:10.1016/j.cyto.2009.02.003 (2009).

20 Cook, M. B. et al. A genome-wide association study of prostate cancer in West African men. Hum Genet 133, 509-521, doi:10.1007/s00439-013-1387-z (2014).

21 Nikolich-Zugich, J. The twilight of immunity: emerging concepts in aging of the immune system. Nat Immunol 19, 10-19, doi:10.1038/s41590-017-0006-x (2018).

663

664

665

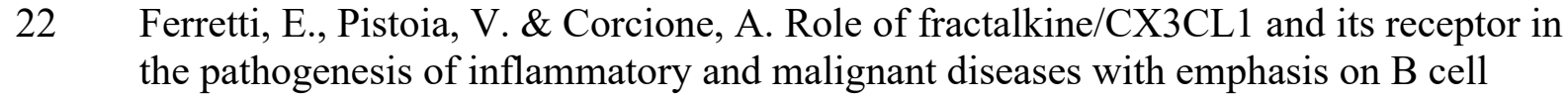
malignancies. Mediators Inflamm 2014, 480941, doi:10.1155/2014/480941 (2014).

670 carbonic anhydrase IX (CA IX) in the serum and urine of renal carcinoma patients. $\mathrm{Br} J$ Cancer 89, 1067-1071, doi:10.1038/sj.bjc.6601264 (2003).

Supuran, C. T. Carbonic anhydrases: novel therapeutic applications for inhibitors and activators. Nat Rev Drug Discov 7, 168-181, doi:10.1038/nrd2467 (2008).

25 Erra Diaz, F., Dantas, E. \& Geffner, J. Unravelling the Interplay between Extracellular Acidosis and Immune Cells. Mediators Inflamm 2018, 1218297 , doi:10.1155/2018/1218297 (2018).

26 Alessandrini, F., Pezze, L. \& Ciribilli, Y. LAMPs: Shedding light on cancer biology. Semin Oncol 44, 239-253, doi:10.1053/j.seminoncol.2017.10.013 (2017).

27 Rabinovich, G. A. \& Toscano, M. A. Turning 'sweet' on immunity: galectin-glycan interactions in immune tolerance and inflammation. Nat Rev Immunol 9, 338-352, doi:10.1038/nri2536 (2009).

28 Arnson, Y., Shoenfeld, Y. \& Amital, H. Effects of tobacco smoke on immunity, inflammation and autoimmunity. J Autoimmun 34, J258-265, doi:10.1016/j.jaut.2009.12.003 (2010).

29 Wada, J. \& Makino, H. Innate immunity in diabetes and diabetic nephropathy. Nat Rev Nephrol 12, 13-26, doi:10.1038/nrneph.2015.175 (2016).

30 Lee, Y. S. et al. The fractalkine/CX3CR1 system regulates beta cell function and insulin secretion. Cell 153, 413-425, doi:10.1016/j.cell.2013.03.001 (2013).

31 Shah, R. et al. Fractalkine is a novel human adipochemokine associated with type 2 diabetes. Diabetes 60, 1512-1518, doi:10.2337/db10-0956 (2011).

32 Navarro-Gonzalez, J. F., Mora-Fernandez, C., Muros de Fuentes, M. \& Garcia-Perez, J. Inflammatory molecules and pathways in the pathogenesis of diabetic nephropathy. Nat Rev Nephrol 7, 327-340, doi:10.1038/nrneph.2011.51 (2011). 
33 Liu, Z. Q. et al. Prognostic role of C-reactive protein in prostate cancer: a systematic review and meta-analysis. Asian J Androl 16, 467-471, doi:10.4103/1008-682X.123686 (2014).

34 Saito, K. \& Kihara, K. C-reactive protein as a biomarker for urological cancers. Nat Rev Urol 8, 659-666, doi:10.1038/nrurol.2011.145 (2011).

35 Al-Alem, U. et al. Association of genetic ancestry with breast cancer in ethnically diverse women from Chicago. PLoS One 9, e112916, doi:10.1371/journal.pone.0112916 (2014).

36 Rotimi, C. N. et al. The genomic landscape of African populations in health and disease. Hum Mol Genet 26, R225-R236, doi:10.1093/hmg/ddx253 (2017).

37 Barreiro, L. B. \& Quintana-Murci, L. From evolutionary genetics to human immunology: how selection shapes host defence genes. Nat Rev Genet 11, 17-30, doi:10.1038/nrg2698 (2010).

38 Gardner, L., Patterson, A. M., Ashton, B. A., Stone, M. A. \& Middleton, J. The human Duffy antigen binds selected inflammatory but not homeostatic chemokines. Biochem Biophys Res Commun 321, 306-312, doi:10.1016/j.bbrc.2004.06.146 (2004).

39 Mantovani, A., Bonecchi, R. \& Locati, M. Tuning inflammation and immunity by chemokine sequestration: decoys and more. Nat Rev Immunol 6, 907-918, doi:10.1038/nri1964 (2006).

40 Miller, L. H., Mason, S. J., Clyde, D. F. \& McGinniss, M. H. The resistance factor to Plasmodium vivax in blacks. The Duffy-blood-group genotype, FyFy. N Engl J Med 295, 302-304, doi:10.1056/NEJM197608052950602 (1976).

41 Jenkins, B. D. et al. Atypical Chemokine Receptor 1 (DARC/ACKR1) in Breast Tumors Is Associated with Survival, Circulating Chemokines, Tumor-Infiltrating Immune Cells, and African Ancestry. Cancer Epidemiol Biomarkers Prev 28, 690-700, doi:10.1158/1055-9965.EPI-18-0955 (2019).

42 Martini, R. et al. Investigation of Triple-Negative Breast Cancer Risk Alleles in An International African-Enriched Cohort. scientific reports Preprint at: https://www.researchsquare.com/article/rs-109841/v1 (2021).

43 Yao, S. et al. Genetic ancestry and population differences in levels of inflammatory cytokines in women: Role for evolutionary selection and environmental factors. PLoS Genet 14, e1007368, doi:10.1371/journal.pgen.1007368 (2018).

44 Strieter, R. M. et al. The functional role of the ELR motif in CXC chemokine-mediated angiogenesis. J Biol Chem 270, 27348-27357, doi:10.1074/jbc.270.45.27348 (1995).

45 Bikfalvi, A. \& Billottet, C. The CC and CXC chemokines: major regulators of tumor progression and the tumor microenvironment. Am J Physiol Cell Physiol 318, C542C554, doi:10.1152/ajpcell.00378.2019 (2020).

46 Calagua, C. et al. Expression of PD-L1 in Hormone-naive and Treated Prostate Cancer Patients Receiving Neoadjuvant Abiraterone Acetate plus Prednisone and Leuprolide. Clin Cancer Res 23, 6812-6822, doi:10.1158/1078-0432.CCR-17-0807 (2017).

47 Awasthi, S. et al. Comparative Genomics Reveals Distinct Immune-oncologic Pathways in African American Men with Prostate Cancer. Clin Cancer Res 27, 320-329, doi:10.1158/1078-0432.CCR-20-2925 (2021).

48 Sartor, O. et al. Survival of African-American and Caucasian men after sipuleucel-T immunotherapy: outcomes from the PROCEED registry. Prostate Cancer Prostatic Dis 23, 517-526, doi:10.1038/s41391-020-0213-7 (2020). 
768

769

770

771

772

773

774

775

49 Segal, N. H. et al. Phase I Study of Single-Agent Utomilumab (PF-05082566), a 41BB/CD137 Agonist, in Patients with Advanced Cancer. Clin Cancer Res 24, 1816-1823, doi:10.1158/1078-0432.CCR-17-1922 (2018).

50 Qi, X. et al. Optimization of 4-1BB antibody for cancer immunotherapy by balancing agonistic strength with FcgammaR affinity. Nat Commun 10, 2141, doi:10.1038/s41467019-10088-1 (2019).

51 Michel, J., Langstein, J., Hofstadter, F. \& Schwarz, H. A soluble form of CD137 (ILA/4$1 \mathrm{BB})$, a member of the TNF receptor family, is released by activated lymphocytes and is detectable in sera of patients with rheumatoid arthritis. Eur J Immunol 28, 290-295, doi:10.1002/(SICI)1521-4141(199801)28:01<290::AID-IMMU290>3.0.CO;2-S (1998).

52 Michel, J. \& Schwarz, H. Expression of soluble CD137 correlates with activationinduced cell death of lymphocytes. Cytokine 12, 742-746, doi:10.1006/cyto.1999.0623 (2000).

53 Labiano, S. et al. Hypoxia-induced soluble CD137 in malignant cells blocks CD137Lcostimulation as an immune escape mechanism. Oncoimmunology 5, e1062967, doi:10.1080/2162402X.2015.1062967 (2016).

54 Itoh, A. et al. Soluble CD137 Ameliorates Acute Type 1 Diabetes by Inducing T Cell Anergy. Front Immunol 10, 2566, doi:10.3389/fimmu.2019.02566 (2019).

55 Kachapati, K. et al. The B10 Idd9.3 locus mediates accumulation of functionally superior $\mathrm{CD} 137(+)$ regulatory $\mathrm{T}$ cells in the nonobese diabetic type 1 diabetes model. $J$ Immunol 189, 5001-5015, doi:10.4049/jimmunol.1101013 (2012).

56 Freeman, Z. T. et al. A conserved intratumoral regulatory T cell signature identifies 4$1 \mathrm{BB}$ as a pan-cancer target. $J$ Clin Invest 130, 1405-1416, doi:10.1172/JCI128672 (2020).

57 Papadimitriou, E. et al. Pleiotrophin and its receptor protein tyrosine phosphatase beta/zeta as regulators of angiogenesis and cancer. Biochim Biophys Acta 1866, 252-265, doi:10.1016/j.bbcan.2016.09.007 (2016).

58 Liu, S. et al. Discovery of PTN as a serum-based biomarker of pro-metastatic prostate cancer. Br J Cancer 124, 896-900, doi:10.1038/s41416-020-01200-0 (2021).

59 Minas, T. Z. et al. IFNL4-DeltaG is associated with prostate cancer among men at increased risk of sexually transmitted infections. Commun Biol 1, 191, doi:10.1038/s42003-018-0193-5 (2018).

60 Network, N. C. C. The NCCN Clinical Practice Guidelines in Oncology for Prostate Cancer, V4.2019.

https://www.nccn.org/professionals/physician gls/default.aspx\#prostate (Accessed November 16, 2020).

61 Candia, J. \& Tsang, J. S. eNetXplorer: an R package for the quantitative exploration of elastic net families for generalized linear models. BMC Bioinformatics 20, 189, doi:10.1186/s12859-019-2778-5 (2019). 
Fig. 1

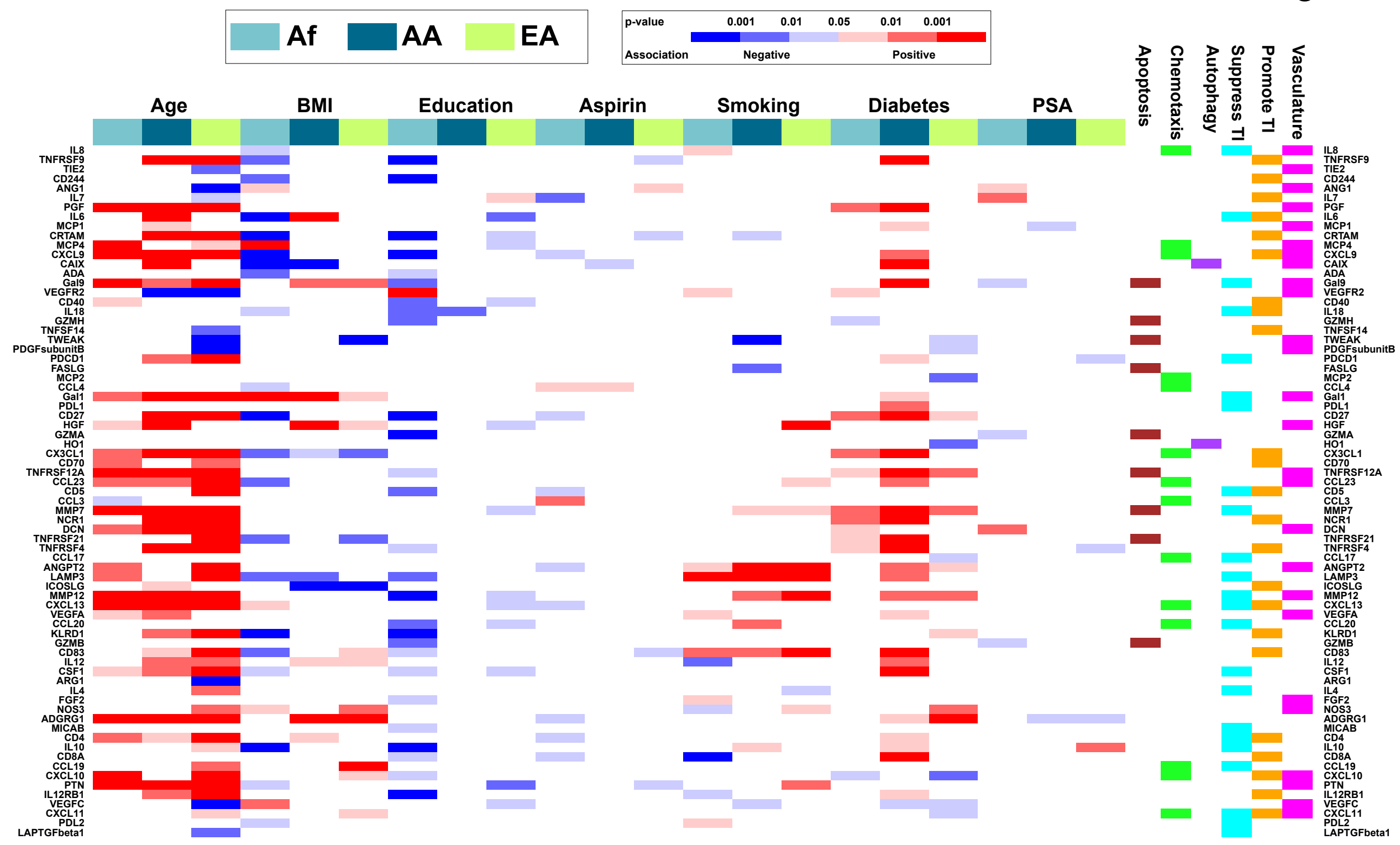

Fig. 1. Association of socio-demographic and clinical characteristics with systemic immune-oncological proteins in Ghanaian (Af), AA, and EA men without prostate cancer. Association of the 82 immuno-oncological proteins with age, BMI, education, aspirin use, smoking, diabetes, and PSA was assessed in men without prostate cancer using a multivariable linear regression model. An analyte was considered significantly associated with clinical and socio-demographic covariables if the multivariable model yielded a $P<0.05$ on the F-statistic. Analytes that did not have a significant association with any of the clinical/sociodemographic variables in at least one of the population groups are not presented in the heatmap. Blue represents negative association while red represents positive association. The significance level ( $P$ value-based) for each association is color-coded. $\mathrm{TI}=$ tumor immunity. 
Fig. 2

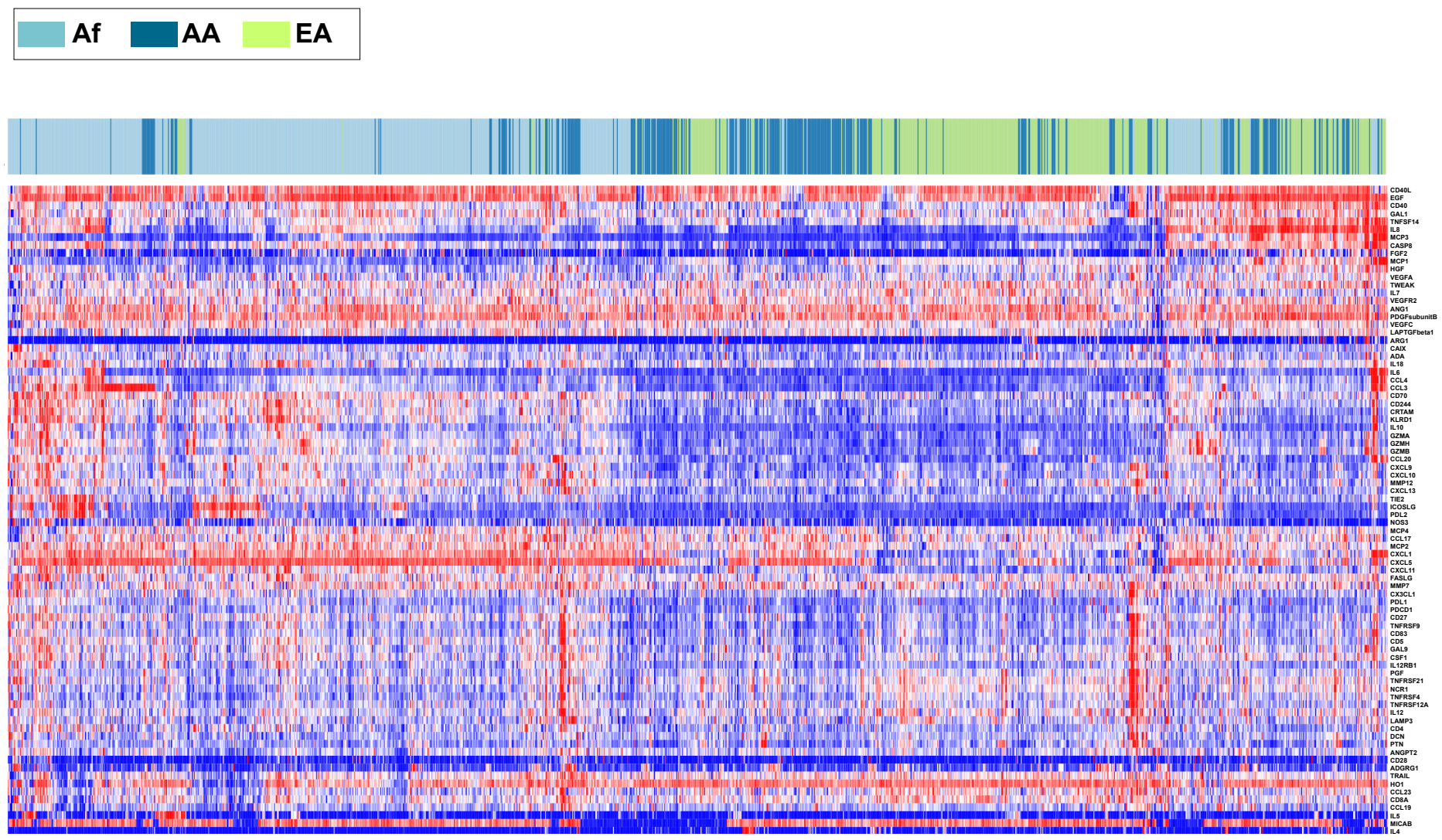

Fig. 2. Unsupervised hierarchical clustering associates circulating immune-oncological proteome profiles with population groups - Ghanaian (Af), AA, and EA men. Heatmap showing protein profiles for men without prostate cancer. Each row represents a protein $(n=82)$, and each column corresponds to an individual [n=1482 (654 Af, $374 \mathrm{AA}$, and $454 \mathrm{EA}$ )]. Each individual is color-coded as Af, AA, or EA in the annotation bar on top of the heatmap. Normalized z-score of proteins abundance are depicted on a low-to-high scale (blue-white-red). 


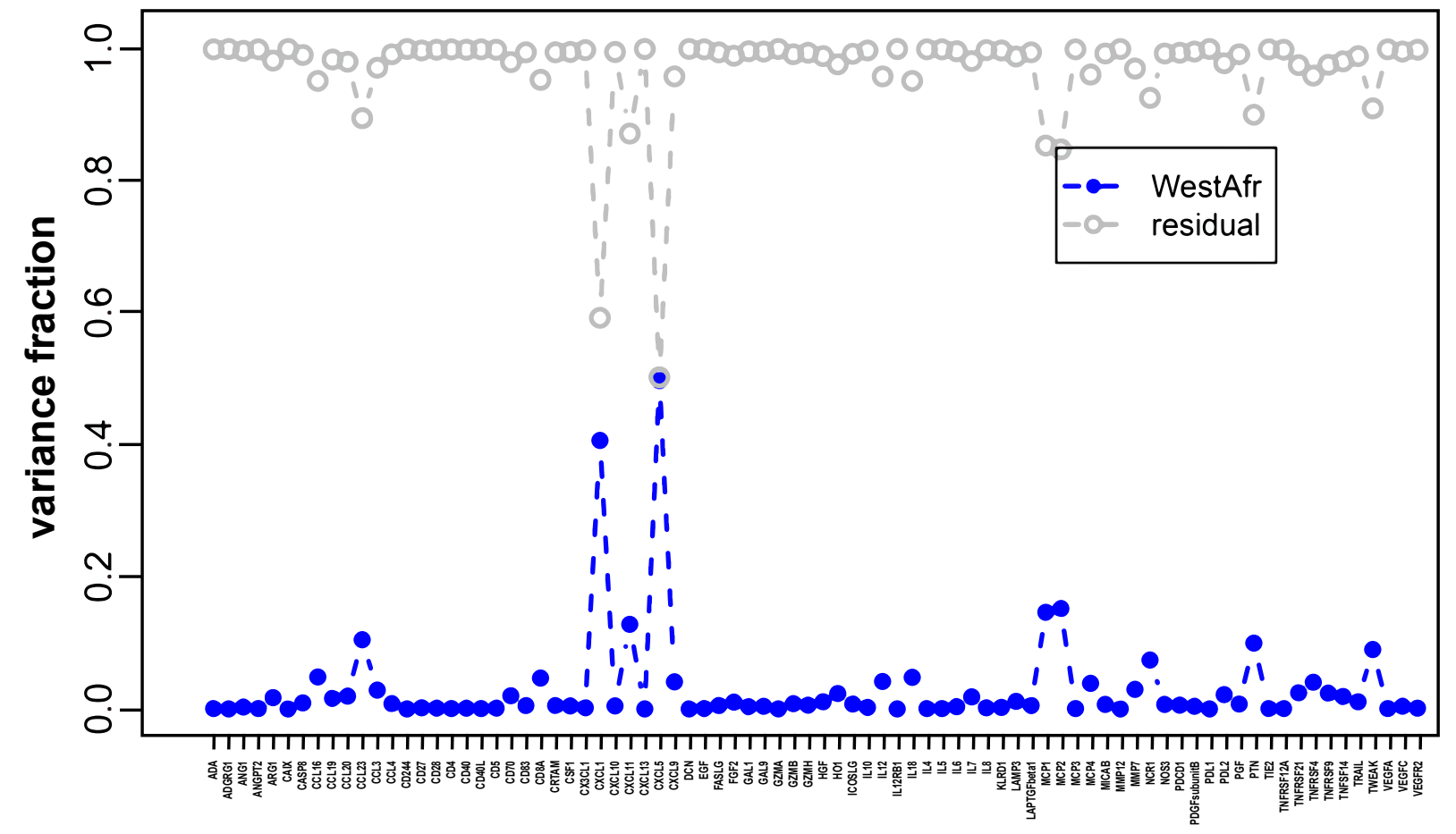

B)
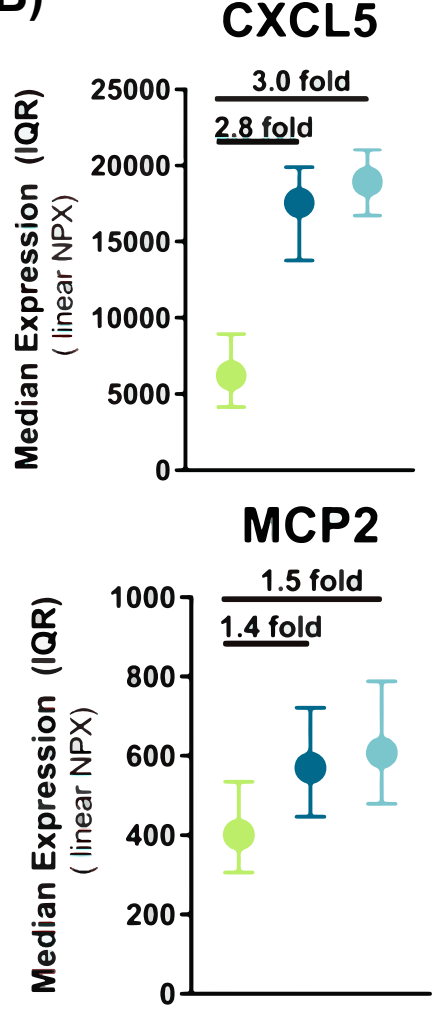

CXCL1

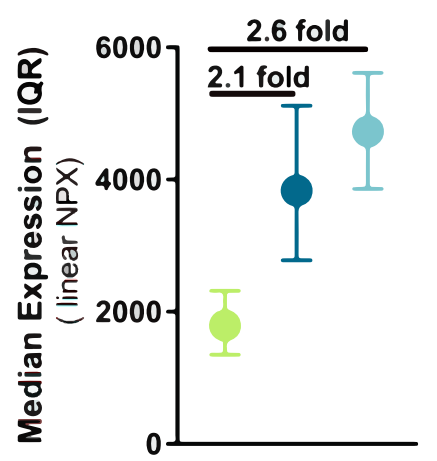

MCP1

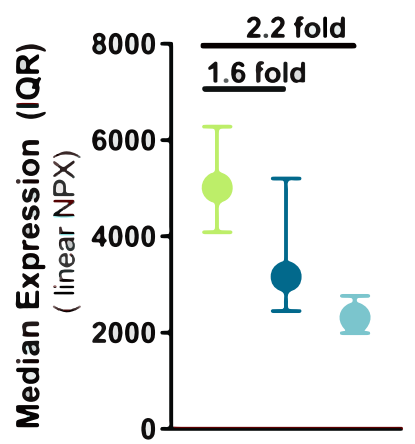

CXCL11

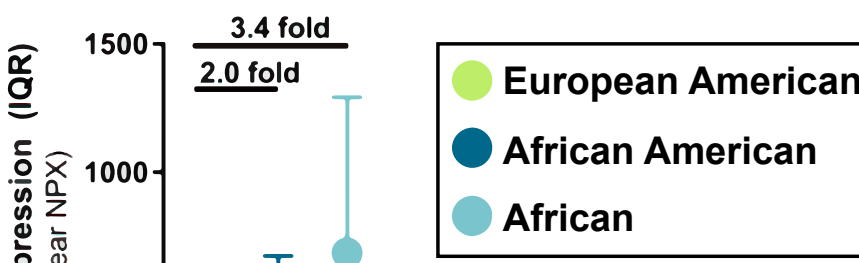

Fig. 3. Immune-oncological proteins and their relationship with West-African ancestry. (A) Variance analysis for the levels of each of the 82 immune-oncological cytokines assessed as a function of genetic estimation of West African admixture among men without prostate cancer within the NCl-Maryland study. The blue plot represents the proportion of variance that can be explained by the degree of West-African admixture while the grey plot represents the residual variance that remains to be explained by other factors other than West-African ancestry. (B) The median levels of the top six West-African ancestry correlated immune-oncological proteins were compared between Af, AA, and EA. Error bars represent inter quartile range (IQR). Linearized protein abundances $\left(2^{\mathrm{NPX}}\right)$ were used to determine median and IQR for each of the proteins. 


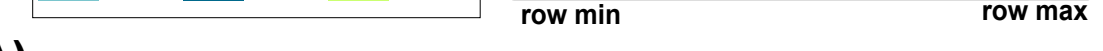

A)

EA

row min

Autophagy

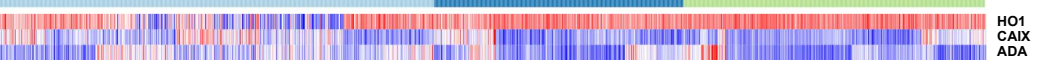

B)

\section{Apoptosis}

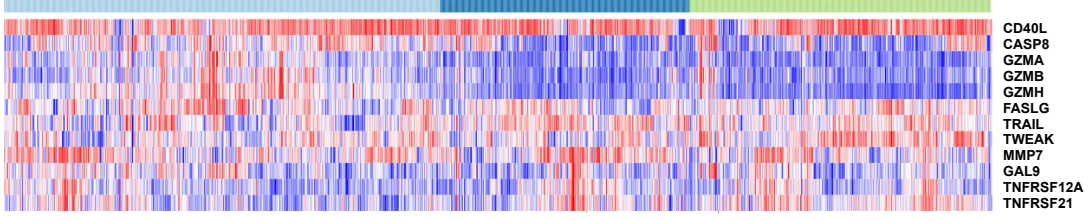

C)

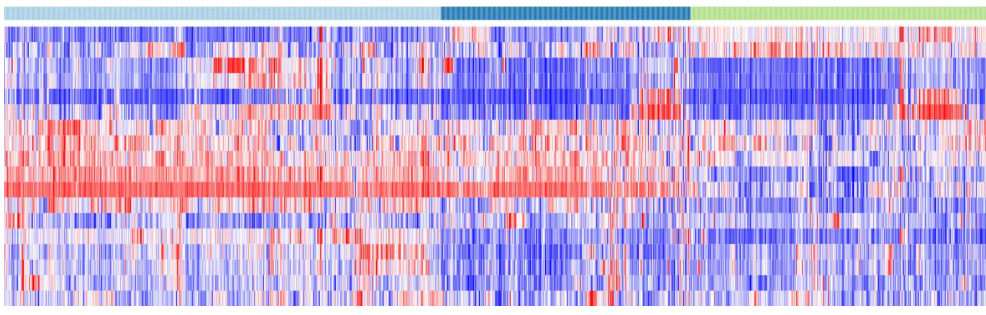

D)

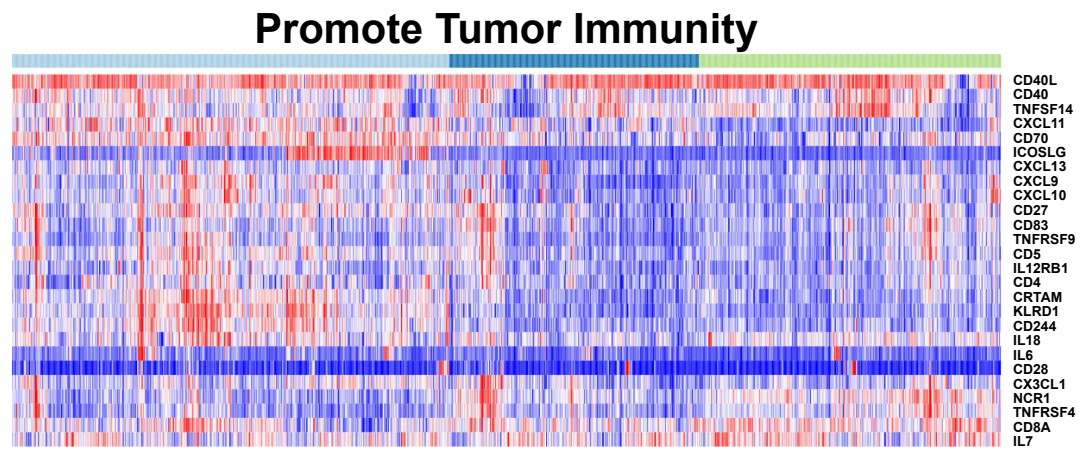

E)

\section{Suppress Tumor Immunity}

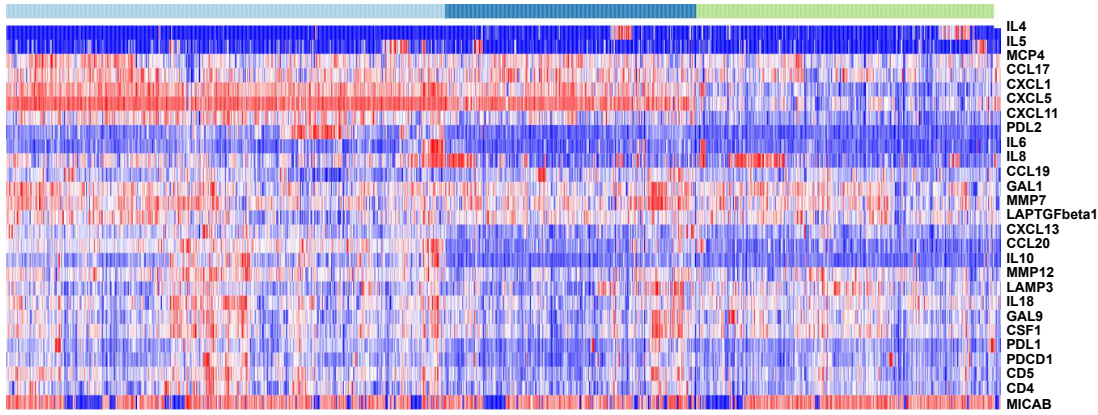

F)

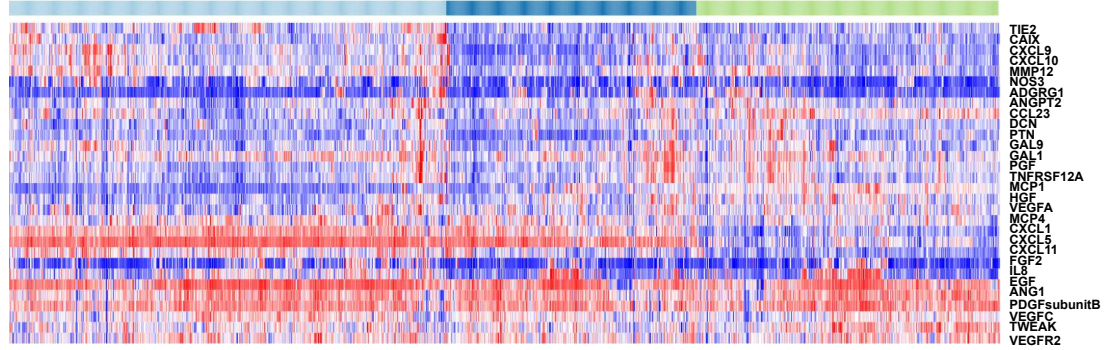

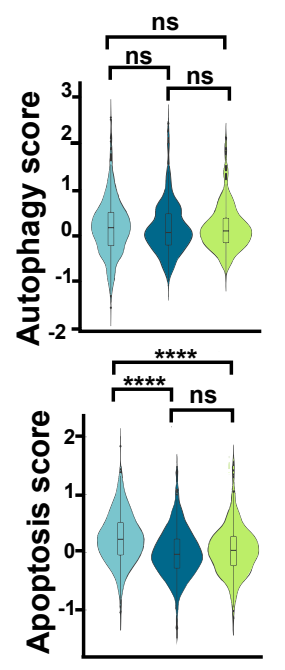

Fig. 4
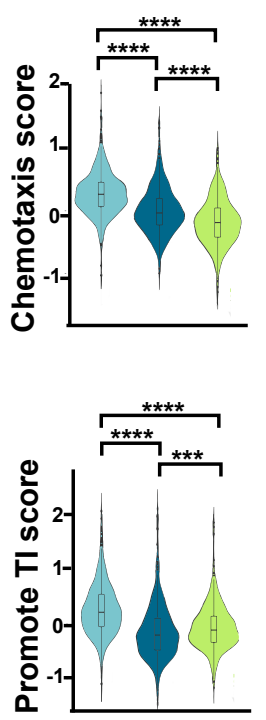

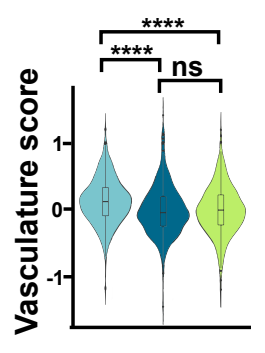

Fig. 4. Population differences in the abundance of proteins driving (A) apoptosis, (B) autophagy, (C) chemotaxis, (D) promotion of tumor immunity, (E) suppression of tumor immunity, and (F) vasculature. Heatmaps showing levels of process/pathway-associated proteins in relationship to population group [Ghanaian (Af), AA, EA]. Shown to the right are the mean score differences for these processes/pathways among the three population groups. Profiles for Ghanaian ( $n=654), A A(n=374)$, and EA ( $n=454)$ men without prostate cancer. The process/pathway scores are derived from the average Z-scores of all the associated proteins. These scores are shown as violin plots. 


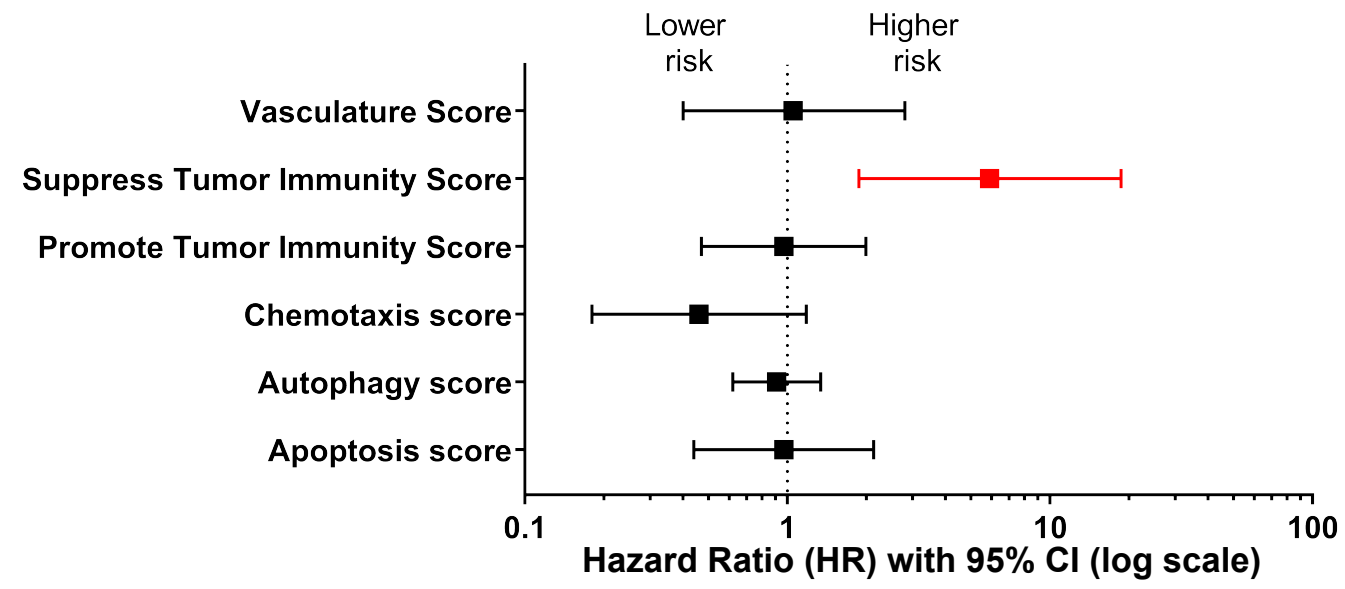

B)

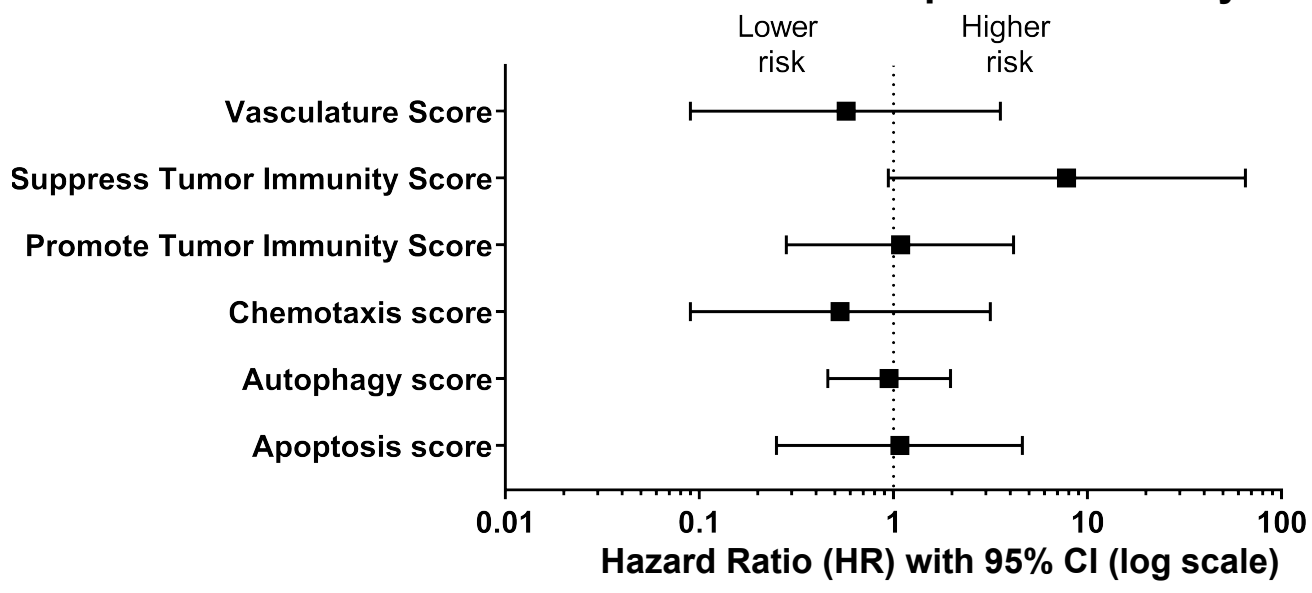

C)
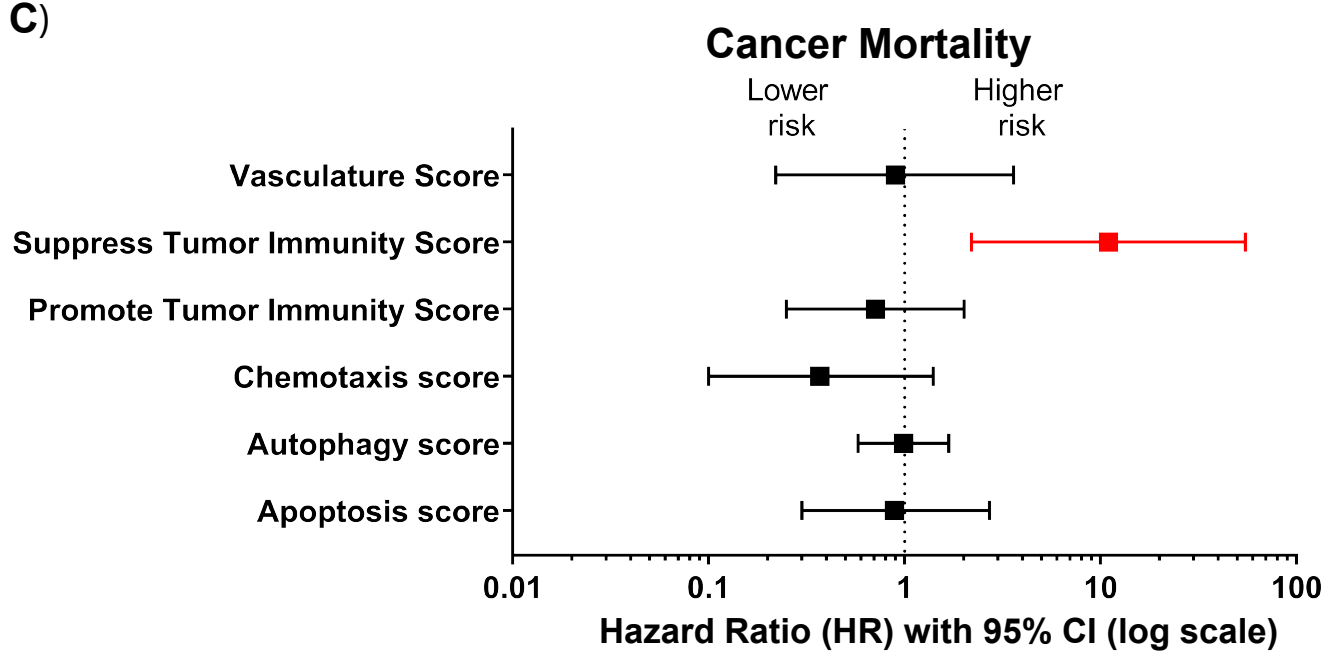

Fig. 5. Suppression of the tumor immunity pathway associates with lethal prostate cancer. We assessed the association of the six pathways defined by the 82 immune-oncology markers with all-cause mortality, prostate cancer-specific mortality or mortality due to any cancer after a prostate cancer diagnosis. The pathway scores were evaluated as continuous predictor variables. Suppression of tumor immunity pathway was distinctively associated with all-cause mortality (A), prostate cancer-specific mortality (B), or a mortality due to any cancer after a prostate cancer diagnosis (C). Multivariable cox regression analyses were used to assess if the pathways were independently associated with survival of prostate cancer patients in the NCl-Maryland study. We adjusted for the following potential confounding factors: age at study entry (years), body-mass index (BMI, $\mathrm{kg} / \mathrm{m} 2$ ), self-reported race (AA/EA), education (high school or less, some college, college, professional school), income (less than $\$ 10 k, \$ 10-30 K, \$ 30-60 K, \$ 60-90 k$, greater than $\$ 90 k)$, smoking history (never, former, current), diabetes (no/yes), aspirin use (no/yes), and treatment ( $0=$ none, $1=$ surgery, 2 =radiotherapy, $3=$ hormone, 4=combination). The hazard ratios (HR) indicate the change in risk of dying when the biological process z-score value increases by 1 while holding all the other biological processes' z-scores and covariates constant. 
A) Top predictors selected based on feature frequency

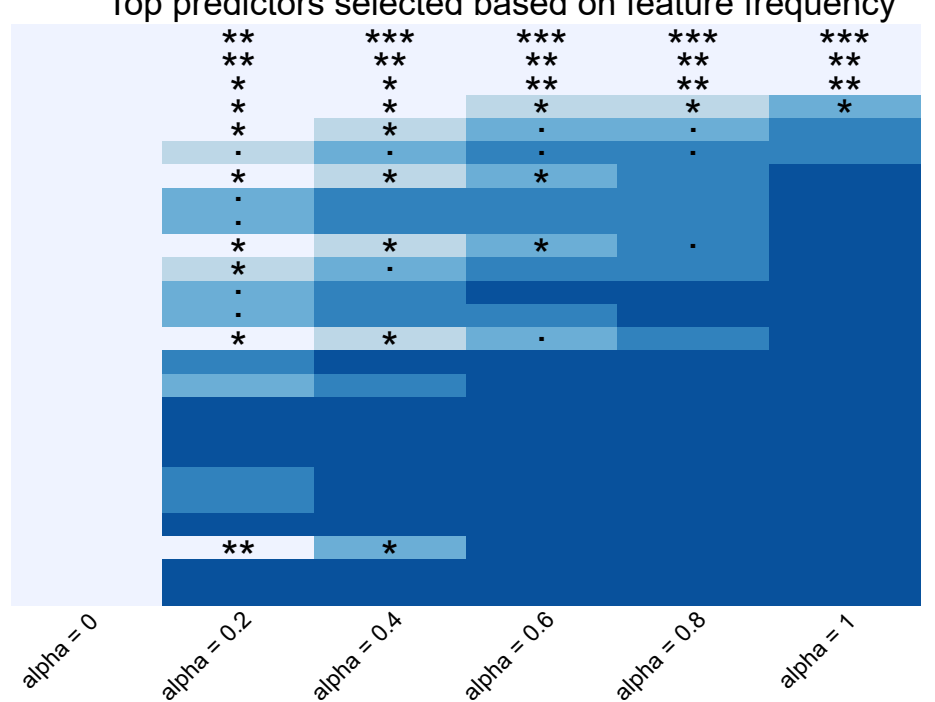

TNFRSF9
PTN

aspirin

aspirin
ADA

bmi

VEGFR2

CD4

PDGFsubunitB

FGF2

MMP7

KLRD1

CXCL1

CXCL10

LAPTGFbeta1

LAPTGF
CD8A

GD8A

CD70

IL7

GZMA

CXCL11

VEGFC

education

CCL23

B) Top predictors selected based on feature coefficients

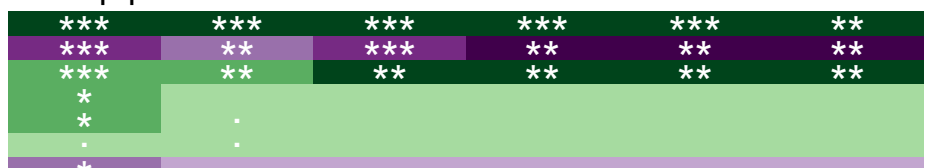

PTN
aspirin

aspirin
TNFRSF9

ADA

MMP7

CD4

bmi

VEGFR2

age

GZMB

CD70

GZMA

PDGFsubunitB

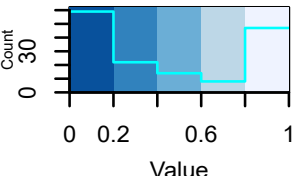

Fig. 6

CD8A

CXCL10

KLRD1

education

FGF2

LAPTGFbeta1

VEGFC

CXCL1

IL7

CCL23

CXCL11

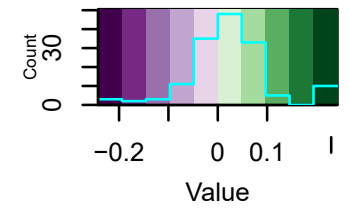

C)
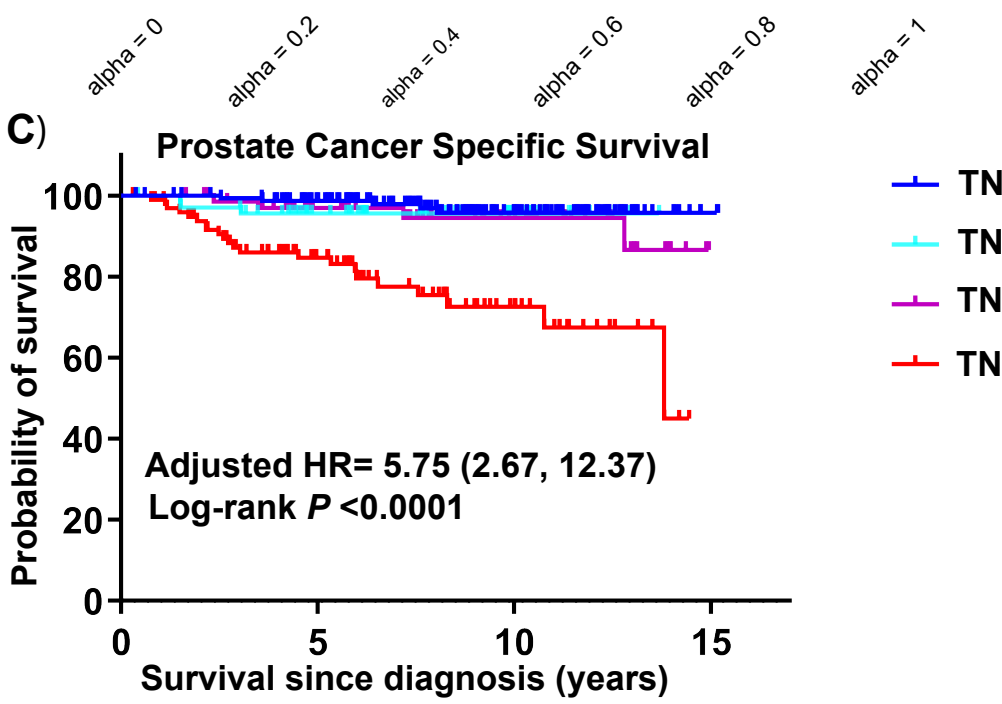

- TNFRSF9 low + PTN low, $(n=159)$

- TNFRSF9 high + PTN low, $(n=69)$

- TNFRSF9 low + PTN high, $(n=68)$

- TNFRSF9 high + PTN high, $(n=98)$

Fig. 6. A signature of two serum markers is predictive of lethal prostate cancer in AA patients. Cross-validated, regularized Cox regression models with different elastic net mixture parameters from ridge (alpha $=0$ ) to lasso $(a l p h a=1)$ were implemented to identify a predictive proteomic signature. (A) Heatmaps of feature frequencies across alpha. Features were ranked by $P$ value for alpha=1. (B) Heatmaps of feature coefficients across alpha. Features were ranked by $P$ value for alpha=1. (C) Kaplan-Meier plot comparing prostate cancer-specific mortality of AA cases with high levels (> median) of both TNFRSF9 and PTN (pleiotrophin) vs. low levels of either or both proteins. Log rank test was used to determine if there were statistically significant survival differences. Adjusted hazard ratio (HR) compares the risk of prostate cancer mortality for those with high levels of both TNFRSF9 and PTN vs. the remaining AA cases. HR estimates were adjusted for potential confounding factors: age, BMI, education, smoking history, diabetes status, aspirin use, treatment, and income. In B \& C, P value significance was coded as $<0.001\left({ }^{* * *}\right),<0.01\left({ }^{* *}\right),<0.05\left(^{*}\right)$, and $<0.1($.). 


\begin{tabular}{|c|c|c|c|}
\hline NCCN Risk Score & $\begin{array}{c}\text { Total } \\
\text { OR }(95 \% \mathrm{Cl}) *\end{array}$ & $\begin{array}{c}\text { AA } \\
\text { OR }(95 \% \mathrm{Cl}) *\end{array}$ & $\begin{array}{c}\text { EA } \\
\text { OR }(95 \% \mathrm{Cl}) *\end{array}$ \\
\hline Low & Ref & Ref & Ref \\
\hline Intermediate & $1.04(0.68-1.59)$ & $0.89(0.46-1.70)$ & $1.18(0.65,2.13)$ \\
\hline High/Very High & $1.47(0.87-2.48)$ & $1.33(0.59-2.98)$ & $1.72(0.83,3.54)$ \\
\hline Regional/Metastatic & 3.79 (1.59-9.04) & $5.90(1.43-24.34)$ & $3.16(0.95,10.50)$ \\
\hline$P$ value for trend & 0.004 & 0.019 & 0.040 \\
\hline \multicolumn{4}{|c|}{$\begin{array}{l}\text { Note: Bolded data indicate significant associations in the logistic regression analysis. } \\
\text { *Logistic regression adjusted for age at study entry, BMI }(\mathrm{kg} / \mathrm{m} 2) \text {, diabetes (no/yes), aspirin } \\
\text { (no/yes), education (high school or less, some college, college, professional school), family } \\
\text { history of prostate cancer (first-degree relatives, yes } / \mathrm{no}) \text {, self-reported race (not included in } \\
\text { the stratified analysis), income (less than } \$ 10 \mathrm{k}, \$ 10-30 \mathrm{~K}, \$ 30-60 \mathrm{~K}, \$ 60-90 \mathrm{k} \text {, greater than } \\
\$ 90 \mathrm{k}) \text {, smoking history (never, former, current), treatment ( } 0=\text { none, } 1=\text { surgery, } 2=\text { radiation, } \\
3=\text { hormone, } 4=\text { combination) }\end{array}$} \\
\hline \multicolumn{4}{|c|}{$\begin{array}{l}\text { High suppression of tumor immunity is defined by the median score in the NCI-Maryland } \\
\text { control population (> median vs. } \leq \text { median) }\end{array}$} \\
\hline
\end{tabular}




\section{Supplementary Files}

This is a list of supplementary files associated with this preprint. Click to download.

- SupplementalMaterial.pdf

- CXUsersIdq5835DesktopSupplementaryTable14.xIsx 